\title{
LE CORTI TEDESCHE ED IL REGOLAMENTO (CE) N. 4/2009 SULLE OBBLIGAZIONI ALIMENTARI
}

\section{GERMAN COURTS AND THE REGULATION (EC) N. 4/2009 ON MAINTENANCE OBLIGATIONS}

\author{
Giovanna Ricciardi \\ Dottoranda di ricerca in Diritto dell'Unione europea presso l'Università \\ degli Studi di Milano-Bicocca
}

Recibido: 14.12.2020 / Aceptado: 14.01.2021

DOI: https://doi.org/10.20318/cdt.2021.5973

\begin{abstract}
Riassunto: Il presente studio offre una panoramica sull'applicazione da parte delle corti tedesche del Regolamento (CE) n. 4/2009 del Consiglio del 18 dicembre 2008 relativo alla competenza, alla legge applicabile, al riconoscimento ed esecuzione delle decisioni e alla cooperazione in materia di obbligazioni alimentari e del Protocollo dell'Aia del 23 novembre 2007 relativo alla legge applicabile alle obbligazioni alimentari.

Lo studio analizza le pronunce più significative relative ai profili di maggiore criticità nell' operatività di questi strumenti a (quasi) dieci anni dalla loro applicazione nell'Unione europea.

Parole chiave: Reg. n. 4/2009, competenza internazionale, legge applicabile, riconoscimento ed esecuzione delle decisioni, Protocollo dell'Aia del 2007.

Abstract: The paper offers an overview on the application in the German courts of the Council Regulation (EC) n. 4/2009 of 18 December 2008 on jurisdiction, applicable law, recognition and enforcement of decisions and cooperation in matters relating to maintenance obligations and the Hague Protocol of 23 November 2007 on the Law Applicable to Maintenance Obligations.

The study analyses those judgments portraying the most significant difficulties of employment of these legal instruments almost ten years after their application in the European Union.

Keywords: Regulation (EC) n. 4/2009, jurisdiction, applicable law, recognition and enforcement of decisions, 2007 Hague Protocol.

Sommario: I. Introduzione. II. La competenza giurisdizionale. III. L'electio fori e la proroga tacita di competenza. IV. La competenza sussidiaria ed il forum necessitatis. V. La giurisdizione per gli enti pubblici. VI. Il Protocollo dell'Aia del 2007. VII. Il coordinamento tra le fonti sulla legge applicabile. VIII. Il riconoscimento, l'esecutività e l'esecuzione dei provvedimenti in materia alimentare. IX. Le disposizioni transitorie.
\end{abstract}

\section{Introduzione}

1. Il Regolamento (CE) n. 4/2009 del 18 dicembre 2008 relativo alla competenza, alla legge applicabile e all'esecuzione delle decisioni e alla cooperazione in materia di obbligazioni alimentari ${ }^{1}$ si applica dal 18 giugno 2011 in tutti gli Stati membri dell'Unione europea, ad eccezione del Regno di

${ }^{1}$ In G.U.U.E. n. L 7 del 10.1.2009, in seguito Reg. n. 4/2009 o regolamento. 
Danimarca. ${ }^{2}$ Questo strumento, nato dall'esigenza di una disciplina organica in tema di obbligazioni alimentari, si inserisce nel processo di "comunitarizzazione" del diritto internazionale privato di famiglia e mira ad assicurare la rapida circolazione delle decisioni in questo settore. La disciplina della legge applicabile è invece soggetta, per espresso rinvio del regolamento stesso, al Protocollo dell'Aia del 2007 sulla legge applicabile alle obbligazioni alimentari.

Esso è entrato in vigore sul piano internazionale il $1^{\circ}$ agosto 2013; tuttavia gli Stati dell'Unione europea ne hanno disposto, limitatamente ai soli rapporti interni, ${ }^{3}$ un'applicazione anticipata al 18 giugno 2011, ${ }^{4}$ per fare coincidere l'applicazione del Protocollo con quella del Reg. n. 4/2009.

2. L'ambito di applicazione ratione materiae del Reg. n. 4/2009 concerne, come indicato all'art. 1 , tutte «le obbligazioni alimentari derivanti da rapporti di famiglia, di parentela, di matrimonio o di affinità». È dunque esclusa l'applicazione della disciplina de qua alle prestazioni risarcitorie, successorie o concernenti il regime patrimoniale tra coniugi. ${ }^{6}$

Tuttavia il regolamento non offre una definizione della nozione di "obbligazione alimentare", la quale per espressa indicazione del considerando n. 11, deve essere interpretata «in maniera autonoma». ${ }^{7}$ Questa soluzione è stata adottata al fine di evitare che il creditore subisca un trattamento differente a seconda del giudice di fronte al quale agisca, in quanto il concetto di "obbligazione alimentare" assume un significato diverso in ciascuno Stato membro. Per comprenderne quindi la portata, ci si deve riferire alle pronunce della Corte di giustizia dell'Unione europea ${ }^{8}$ in vigenza degli strumenti che hanno preceduto il regolamento, ${ }^{9}$ coi quali sussiste un rapporto di continuità. ${ }^{10}$ Pertanto, la nozione di obbligazione

\footnotetext{
${ }^{2}$ Il Regno di Danimarca, pur non partecipando al Reg. n. 4/2009, con lettera del 14 gennaio 2009 ha notificato alla Commissione la decisione di attuare il contenuto dello stesso nella misura in cui modifichi il Regolamento (CE) n. 44/2001 (cd. Reg. Bruxelles I). Di questa si dà atto nella Comunicazione della Commissione Accordo tra la Comunità europea e il Regno di Danimarca concernente la competenza giurisdizionale, il riconoscimento e l'esecuzione delle decisioni in materia civile e commerciale, in G.U.U.E. n. L 149 del 12.6.2009.

${ }^{3}$ Sulla posizione del Regno di Danimarca e Regno Unito cfr. Protocollo (N.22) sulla posizione della Danimarca allegato al TFUE, in G.U.U.E. n. C 326 del 26.10.2012 e Protocollo (N.21) sulla posizione del Regno Unito e dell'Irlanda rispetto allo spazio di libertà, sicurezza e giustizia allegato al TFUE, in G.U.U.E. n. C 326 del 26.10.2012.

${ }^{4}$ A nome di tutti gli Stati Membri, in data 8 aprile 2010 l'Unione europea ha firmato il Protocollo dell'Aia del $2007 \mathrm{cfr}$. Decisione del Consiglio 2009/941/CE del 30 novembre 2009, relativa alla conclusione da parte della Comunità europea del protocollo dell'Aia, del 23 novembre 2007, sulla legge applicabile alle obbligazioni alimentari, in G.U.U.E. n. L 331 del 16.12.2009.

${ }^{5}$ F. C. VILlata, «Obblighi alimentari e rapporti di famiglia secondo il regolamento n. 4/2009», in Rivista di diritto internazionale, 2011, p. 734-735; M. CASTELlanETA, A. LEANDRO, «Il regolamento CE n. 4/2009 relativo alle obbligazioni alimentari», in Le nuove leggi civili commentate, 2009, p. 1051-1052.

${ }^{6}$ F. PESCE, Le obbligazioni alimentari tra diritto internazionale e diritto dell'Unione europea, Roma, Aracne 2013, p. 6872; F. C. Villata, ivi, p. 740-742; M. E. CorraO, «Il diritto internazionale privato e processuale europeo in materia di obbligazioni alimentari», in Cuadernos de Derecho Transnacional, 2011, p. 123-124.

${ }^{7}$ L'«interpretazione autonoma» delle nozioni di diritto dell'Unione europea rappresenta il modello prediletto dalla Corte di giustizia. Questo metodo svincola il diritto dell'Unione europea dagli schemi interpretativi nazionali e ne garantisce anche l'applicazione uniforme in tutti gli Stati membri.

${ }^{8}$ Si richiamano, in particolare, Corte di giustizia 6 marzo 1980, De Cavel c. De Cavel, 120/79 e Corte di giustizia 27 febbraio 1997, Van den Boogard c. Laumen. 220/95. In quest'ultima, il giudice comunitario era stato chiamato a fornire una definizione del concetto di "obbligazione alimentare" richiamato nel criterio giurisdizionale dell'art. 5 n. 2 Convenzione di Bruxelles del 1968 (poi ripreso allo stesso articolo dal Reg. n. 44/2001). Ne era scaturita un'interpretazione piuttosto ampia, in quanto nel concetto vi rientravano tutte le obbligazioni finalizzate al sostentamento del creditore, determinate dal giudice o ex lege.

${ }^{9} \mathrm{Ci}$ si riferisce in particolare alla Convenzione di Bruxelles del 1968 concernente la competenza giurisdizionale e l'esecuzione delle decisioni in materia civile e commerciale, resa esecutiva in Italia con la legge 21 giugno 1971 n. 804, in G.U. n. C 27 del 26.1.1998 ed al Regolamento (CE) n. 44/2001 del Consiglio del 22 dicembre 2000 concernente la competenza giurisdizionale, il riconoscimento e l'esecuzione delle decisioni in materia civile e commerciale, in G.U.U.E. n. L 12 del 16.1.2001. Quest'ultimo è entrato in vigore il $1^{\circ}$ marzo 2002 con l'obiettivo di creare uno spazio giudiziario europeo caratterizzato da uniformi norme di giurisdizione, in grado di ridurre o eliminare gli ostacoli frapposti alla circolazione ed all'esecuzione delle decisioni straniere. Dal 10 gennaio 2015, il Reg. n. 44/2001 è stato sostituito dal Regolamento (UE) 1215/2012.

${ }^{10}$ Questo aspetto viene confermato nelle disposizioni del Reg. n. 4/2009. Il considerando n. 44 fa presente la necessità che il Reg. n. 4/2009 sostituisca le disposizioni del Reg. n. 44/2001 relative alle obbligazioni alimentari e che venga applicato in luogo di questi. Anche 1'art. 75, concernente le disposizioni transitorie, salvo alcune eccezioni (vedi infra par. IX), stabilisce l'applicazione del Reg. n. 4/2009 in luogo del Reg. n. 44/2001 per i procedimenti instaurati a partire dal 18 giugno 2011. Il
} 
alimentare che risulta dall'interpretazione del Reg. n. 4/2009 non coincide con quella adottata dai diversi ordinamenti nazionali in quanto, nella maggior parte dei casi, è più ampia e retta da presupposti distinti. ${ }^{11}$

Il regolamento non definisce neppure i rapporti dai quali scaturisce l'obbligazione alimentare, ma a tale lacuna non si può sopperire ricorrendo alla qualificazione autonoma. Ogni Stato membro ha infatti una visione molto distinta del concetto di "famiglia", inevitabilmente influenzato dalla storia e dalla cultura di ciascun Paese. Per questo motivo è necessario riferirsi alle scelte compiute dai legislatori dei singoli Stati membri, i quali determinano se un certo rapporto può includersi nelle categorie di cui all'art. 1 del regolamento. ${ }^{12}$ Questa soluzione viene confermata dal regolamento stesso, il quale dichiara che l'accertamento del rapporto di famiglia su cui si fonda l'obbligazione alimentare è disciplinato dal diritto interno degli Stati membri (considerando n. 21). ${ }^{13}$

\section{La competenza giurisdizionale}

3. Il primo profilo del presente lavoro concerne l'analisi dell'applicazione delle norme sulla competenza internazionale, quale regolata dal capo II del Reg. n. 4/2009, nelle corti tedesche. Dall'art. 3 all'art. 13 infatti, il regolamento disciplina la ripartizione della competenza in ipotesi di controversie alimentari internazionali, ponendo norme uniformi che prevalgono su quelle nazionali. ${ }^{14}$

Conformemente al considerando n. $12^{15}$ queste norme saranno applicate anche dalle autorità amministrative quando esse adottano provvedimenti in materia alimentare. ${ }^{16}$

L'art. 3 attribuisce la competenza alle autorità giurisdizionali dello Stato (a) di residenza abituale del convenuto, (b) di residenza abituale del creditore, nonché alle autorità competenti a conoscere (c) un'azione relativa allo stato delle persone $\mathrm{o}(\mathrm{d})$ un'azione relativa alla responsabilità genitoriale in ipotesi di accessorietà della questione alimentare e ad esclusione dei casi in cui la competenza sia unicamente fondata sulla cittadinanza di una delle parti. Risulta evidente l'abbandono del titolo di giurisdizione basato sul domicilio, come contemplato nel previgente Reg. n. 44/2001 e tutt'oggi nel Reg. n. 1215/2012, ${ }^{17}$ a favore del criterio della residenza abituale, ormai tradizionale in ambito familiare. ${ }^{18}$ Emerge altresì

Reg. n. 44/2001, come indicato dal suo considerando n. 19, garantisce la continuità con la Convenzione di Bruxelles del 1968.

${ }^{11}$ Nell'ordinamento italiano, ad esempio, il concetto di obbligazione alimentare si presenta da un lato più limitato in virtù dell'esclusione del mantenimento dalla disposizione di cui all'art. 433 c.c., e, dall'altro, più ampio in considerazione dell'inclusione della figura del donatario come potenziale debitore, non presente nel Reg. n. 4/2009 in quanto non collegabile al rapporto familiare cfr. I. VIARENGO, «Sulla disciplina degli obblighi alimentari nella famiglia e dei rapporti patrimoniali tra coniugi», in Rivista di diritto internazionale privato e processuale, 2015, p. 827; F. POCAR, «La disciplina comunitaria della giurisdizione in tema di alimenti: il regolamento 4/2009», in Le nuove competenze comunitarie: obbligazioni alimentari e successioni, a cura di M. C. Baruffi, R. Cafari Panico, Padova, Cedam 2009, p. 5-6.

${ }^{12} \mathrm{Si}$ tratta di una soluzione già adottata nel Protocollo dell'Aia del 2007 (vedi infra par. VI)

${ }^{13} \mathrm{~F}$. Albano, «I concetti di "Obbligazione alimentare e di rapporti di famiglia"», in Obbligazioni alimentari nelle controversie transfrontaliere: il recupero delle obbligazioni alimentari nelle controversie transfrontaliere ai sensi del regolamento 4/2009 del Consiglio, a cura di L. Sangiovanni, Roma, Alpes 2014, p. 39-45; M. Castellaneta, A. Leandro, «Il regolamento CE n. 4/2009» cit., p. 1060-1063; F. PoCAR, I. VIARENGo, «Il regolamento (CE) n. 4/2009 in materia di obbligazioni alimentari», in Rivista di diritto internazionale privato e processuale, 2009, p. 807-810.

${ }^{14}$ I. VIARENGO, ivi, p. 825.

${ }^{15}$ Il quale dispone che: «Al fine di tener conto dei diversi modi di disciplinare le questioni relative alle obbligazioni alimentari negli Stati membri, il presente regolamento dovrebbe applicarsi sia alle decisioni giurisdizionali che alle decisioni emesse da autorità amministrative, purché queste autorità offrano garanzie in particolare circa l'imparzialità e il diritto di audizione delle parti. Tali autorità dovrebbero pertanto applicare tutte le norme del presente regolamento».

${ }^{16}$ F. F. Garau Sobrino, «Las fuentes españolas sobre obligaciones alimenticias. ¿Hacia un Derecho internacional privado extravagante?», in Cuadernos de Derecho Transnacional, 2011, p. 134; F. PoCAR, I. VIARENGO, «Il regolamento (CE) n. 4/2009» cit., ${ }^{\text {p. }} 811$.

${ }^{17}$ Il Regolamento (UE) n. 1215/2012 del Parlamento europeo e del Consiglio, del 12 dicembre 2012, concernente la competenza giurisdizionale, il riconoscimento e l'esecuzione delle decisioni in materia civile e commerciale è entrato in vigore il 10 gennaio 2015 ed ha sostituito il Reg. n. 44/2001, in G.U.U.E. n. L 351 del 20.12.2012. Il Regno di Danimarca, con lettera del 20 dicembre 2012, ha notificato alla Commissione la decisione di attuarne il contenuto; questo regolamento si applica pertanto anche alle relazioni fra l'Unione europea e la Danimarca.

${ }^{18} \mathrm{~L}$ 'apertura al criterio della residenza abituale conferma una tendenza già accolta in seno alle convenzioni dell'Aia, nonché ai regolamenti comunitari tra cui Roma I, II e III. Tale criterio risponde al principio di prossimità ed asseconda le esigenze dei 
chiaramente il principio del favor creditoris, stante la possibilità per il creditore di scegliere se instaurare la controversia nel luogo della propria residenza abituale o in quella del debitore. Viceversa, il debitore potrà agire in un unico foro, senza alcuna possibilità di scelta, in quanto il foro della residenza del convenuto (art. 3 lett. a), coincide con quello della residenza del creditore alimentare (art. 3 lett. b). ${ }^{19}$

Al fine di evitare che il debitore possa trarre vantaggio dalla previsione del suo foro, il regolamento introduce una limitazione alla sua azione processuale.

L'art. 8 dispone infatti che quando il giudice dello Stato di residenza del creditore abbia adottato una decisione, il debitore non possa instaurare una controversia per la modifica o l'ottenimento di una nuova pronuncia sugli alimenti di fronte ad un giudice di uno Stato membro diverso fino a quando il creditore continui ad avere la residenza abituale in quest'ultimo Stato.

La ratio alla base di una tale previsione, che di fatto realizza una sorta di perpetuatio fori, consiste nell'offrire uno strumento di protezione del creditore alimentare al fine di scongiurare comportamenti dilatori del debitore nello spazio giudiziario europeo. ${ }^{20}$ Tuttavia, quando sia il creditore a spostare la sua residenza abituale, torneranno ad operare i principi generali di cui all'art. $3 .{ }^{21}$

4. I criteri generali di competenza di cui all'art. 3 non sembrano avere creato particolari problemi applicativi in Germania.

Qualche difficoltà è invece emersa, specie subito dopo l'entrata in vigore del regolamento, nell'applicazione dell'art. 8, forse causata dalla equivoca interpretazione della portata e della ratio alla base della perpetuatio fori.

In un risalente caso del $2012,{ }^{22}$ una causa per la modifica del mantenimento instaurata di fronte all'autorità tedesca che aveva originariamente disposto tale obbligo, nonostante la residenza abituale del creditore fosse mutata, ha consentito al giudice tedesco di precisare i presupposti per l'operatività dell'art. 8.

Il debitore, residente in Germania, chiede all'Amtsgericht $M$. la modifica della misura del mantenimento dovuto alla creditrice e posto a suo carico in fase di divorzio dalle autorità tedesche. La creditrice, residente in Spagna da diversi anni, contesta la competenza delle autorità tedesche a favore di quelle spagnole. In conformità all'art. 10 Reg. n. 4/2009, ai sensi del quale l'autorità giurisdizionale erroneamente adita per una controversia per la quale non abbia competenza deve dichiarare d'ufficio la propria incompetenza, l'Amtsgericht $M$. si dichiara carente di giurisdizione e rigetta la domanda. Avverso tale sentenza, il debitore propone appello.

Anche il giudice di appello ritiene competenti le autorità giurisdizionali spagnole sulla base di quanto prescritto dall'art. 3 lett. a) e b). Poiché la creditrice risiede abitualmente da oltre dieci anni in Spagna, divenuto il centro delle proprie relazioni familiari, sociali e professionali, trovano applicazione i criteri generali in luogo dell'art. 8.

La decisione è evidentemente corretta, poiché il giudice ribadisce che l'art. 8 non opera per il solo fatto che si agisca per la modifica di un'obbligazione alimentare previamente definita, ma solo quando il creditore «continui a risiedere abitualmente nello Stato in cui è stata emessa la decisione», proteggendolo da qualsiasi tentativo di modifica della statuizione presso un foro differente per mano del debitore. Nell'ipotesi in cui però, come nel caso di specie, il creditore abbia modificato la propria residenza abituale trasferendosi in uno Stato membro differente da quello nel quale sia stata emanata la decisione, la perpetuatio iurisdictionis di cui all'art. 8 non può operare, dovendosi fare riferimento ai criteri generali di cui all'art. 3 lett. a) e b).

\footnotetext{
cittadini di circolazione nell'Unione europea cfr. M. CASTELLANETA, A. LEANDRO, «Il regolamento CE n. 4/2009» cit., p. 1069.

${ }^{19}$ F. C. VILlata, «Obblighi alimentari» cit., p. 745-746.

${ }^{20}$ M. Vargas Gómez-Urrutia, «El cobro internacional de alimentos. Reflejos del dispositivo protector del acreedor de alimentos en las normas del Reglamento 4/2009», in Revista de Derecho de la Unión Europea., 2012, p. 105; F. PoCAR, I. VIARENGO, «Il regolamento (CE) n. 4/2009» cit., p. 818-819.

${ }^{21}$ M. NADEMLEINSKY, «Die neue EU-Unterhaltsverordnung samt dem neuen Haager Unterhaltsprotokoll», in Ehe- und Familienrechtliche Zeitschrift, 2011, p. 131.

${ }^{22}$ OLG Düsseldorf 25 aprile 2012, in Zeitschrift für das gesamte Familienrecht, 2013, p. 55-57.
} 


\section{L'electio fori e la proroga tacita di competenza}

5. Conformemente ad una tendenza sempre più diffusa dal legislatore dell'Unione europea, ${ }^{23} \mathrm{ac}-$ canto a criteri di giurisdizione oggettivi, il Reg. n. 4/2009 ammette la scelta del foro competente. Lo spazio di manovra delle parti è però fortemente limitato. ${ }^{24}$ Oltre alla disposizione di cui all'art. 4 comma 3 , la quale vieta una proroga di competenza quando l'accordo riguardi un minorenne, il regolamento consente la scelta solo quando questa sia in favore di autorità giurisdizionali che presentino un certo collegamento con le parti, seppur limitato nel tempo. L'art. 4 lett. a) e b) consente, ad esempio, di scegliere le autorità giurisdizionali dello Stato membro in cui una delle parti abbia la propria residenza abituale o di cui sia cittadina. Laddove invece le obbligazioni alimentari riguardino coniugi o ex coniugi, la legge ammette che le parti realizzino la concentrazione delle controversie presso il giudice competente in materia matrimoniale (lett. c) i) o si esprimano a favore del giudice del luogo dell'ultima residenza comune per almeno un anno (lett. c) ii). ${ }^{25}$ All'accordo sulla scelta del foro viene, in linea di principio, attribuito carattere esclusivo. L'art. 4 comma 1 par. 3 dispone infatti che: «La competenza conferita dall'accordo è esclusiva, salvo che le parti non dispongano diversamente». Ciò significa che con l'electio fori viene meno la possibilità di applicare gli altri criteri di cui all'art. $3^{26} \mathrm{e}$, pertanto, si garantisce la certezza del diritto. ${ }^{27}$

6. Mentre la norma sulla scelta espressa del foro non ha trovato frequente impiego, maggiore applicazione ha trovato l'art. 5 che attribuisce competenza al giudice davanti al quale il convenuto sia comparso senza averne eccepito l'incompetenza (cd. proroga tacita).

L'art. 5 è un criterio di giurisdizione concorrente ed alternativo rispetto a quelli previsti nelle disposizioni che lo precedono.

Se il convenuto compare senza sollevare eccezione di incompetenza ma limitandosi a chiedere il rigetto della pretesa attorea, la competenza è attribuita ai sensi dell'art. 5. Quest'ultimo non contiene regole uniformi sui termini per sollevare tale eccezione in quanto, trattandosi di norme processuali interne,

${ }^{23}$ La bibliografia in materia è ampia; a titolo meramente indicativo: S. BARIATTI, «Volontà delle parti e internazionalità del rapporto giuridico: alcuni sviluppi recenti nella giurisprudenza della Corte di giustizia sui regolamenti europei in materia di diritto internazionale privato», in Rivista di diritto internazionale privato e processuale, 2019, p. 513-534; C. CALABRESE, «La valorizzazione della volontà delle parti nei regolamenti europei in ambito familiare», in Rivista AIAF, 2019; M. PERTEGÁs SENDER, «New International Rules on Choice of Court Agreements in International Contracts: Hague Convention and Brussels I bis Regulation», in Anuario español de Derecho internacional privado, 2014-2015, p. 111-126; O. FerACI, «L'autonomia della volontà nel diritto internazionale privato dell'unione europea», in Rivista di diritto internazionale, 2013, p. 424-491; F. Marongiu Buonaiuti, Le obbligazioni non contrattuali nel diritto internazionale privato, Milano, Giuffrè 2013; T. VASSALLI DI DACHENHAUSEN, «Qualche considerazione sull'autonomia delle parti nel regolamento comunitario 4/2009 in materia di obbligazioni alimentari», in Diritto del commercio internazionale, 2012, p. 361-370; F. C. VILlaTA, L'attuazione degli accordi di scelta del foro nel regolamento Bruxelles I, Padova, Cedam 2012; I. QuerRolo, Gli accordi sulla competenza giurisdizionale, Padova, Cedam 2000;

${ }^{24}$ M. Castellaneta, A. Leandro, «Il regolamento CE n. 4/2009» cit., p. 1070.

${ }^{25} \mathrm{Da}$ un punto di vista formale, per la validità della scelta del foro è necessaria la forma scritta ad substantiam; in vigenza del Reg. n. 44/2001, la forma scritta si imponeva invece ai soli fini probatori. Ai sensi del Reg. n. 4/2009, l'electio fori deve quindi avvenire per iscritto, sotto forma di «qualsiasi comunicazione elettronica che consenta una registrazione durevole dell'accordo» (art. 4 comma 2).

${ }^{26}$ Il punto è tuttavia controverso. Come nel Reg. n. 44/2001 (art. 23 comma 1), anche nel Reg. n. 4/2009 le parti possono attribuire al foro scelto carattere concorrente. Sulla base della condizione/eccezione «salvo che», il carattere di foro concorrente sembra però dover essere chiaramente espresso nell'accordo. Nel dubbio, al foro eletto deve quindi attribuirsi carattere di esclusività. Peraltro, qualche elemento di ambiguità si desume dall'art. 4 comma 4 che sembra considerare il carattere di foro esclusivo come l'eccezione, e non come la regola. Si legge infatti che: «Se le parti hanno convenuto di attribuire competenza esclusiva alla o alle autorità giurisdizionali di uno Stato parte della $[\ldots]$ "convenzione di Lugano" [...]». La dottrina (M. ANDRAE, «Kapitel II: Zuständigkeit Art 4 EG-UntVO», in Europäisches Zivilprozess- und Kollisionsrecht EuZPR/EuIPR, a cura di T. RAuSCHER, Köeln, Dr. Otto Schmidt 2015, p. 547-548) risolve tale conflitto interpretativo considerando quest'ultima disposizione riferibile solo a casi specifici, nei quali la competenza venga attribuita al giudice di uno Stato parte della Convenzione di Lugano del 2007. Pertanto, in tutti gli altri casi, in assenza di indicazioni circa il carattere esclusivo o meno della proroga, la competenza concorrente di un altro giudice deve essere esclusa. Migliore formulazione sembra avere il Reg. n. 1215/2012 che attribuisce carattere esclusivo alla proroga di competenza, salvo diversa volontà delle parti (art. 25).

${ }^{27} \mathrm{~S}$. MARINO, «Il nuovo regolamento comunitario sulla cooperazione giudiziaria civile in materia di obbligazioni alimentari», in La nuova giurisprudenza civile commentata, 2009, p. 604-605. 
la competenza dell'Unione europea è in linea di principio esclusa; sono infatti le disposizioni nazionali di ciascuno Stato a trovare applicazione. ${ }^{28}$

Diversamente da queste ultime però, la proroga tacita di competenza non limita l'autonomia delle parti coinvolte, potendo trovare applicazione anche nelle controversie concernenti i minori.

La norma sulla proroga tacita non prevede nemmeno forme di tutela della parte debole convenuta a differenza, ad esempio, dell'art. 26 comma 2 Reg. n. 1215/2012 il quale richiede al giudice, prima che si dichiari competente, di verificare l'effettiva conoscenza del diritto di eccepire l'incompetenza da parte del convenuto. L'assenza nel Reg. n. 4/2009 di una tale disposizione contribuisce forse ad uno svolgimento più celere della controversia, ma non garantisce alcuna protezione della parte convenuta, la quale potrebbe essersi costituita senza avvalersi di un avvocato.

7. Alcune pronunce tedesche sull'art. 5 consentono di comprendere meglio il meccanismo della proroga tacita, la quale fonda la competenza di un giudice diverso rispetto a colui cui spetterebbe la giurisdizione secondo i criteri oggettivi predeterminati.

Nel caso deciso dall'Amtsgericht Flensburg, ${ }^{29}$ questi si ritiene competente a ridurre la misura del mantenimento alla creditrice che risiede abitualmente nel Regno di Danimarca. Il debitore, padre di quest'ultima, conviene la figlia in giudizio al fine di ottenere la modifica dell'importo del mantenimento fissato dallo Jugendamt della città di previa residenza della creditrice alimentare. Quest'ultima, che al momento dell'instaurazione della controversia si trova temporaneamente in Germania presso la madre, risiede stabilmente nel Regno di Danimarca. Notificato l'atto introduttivo alla creditrice, questa si costituisce in giudizio chiedendo il rigetto della pretesa attorea senza contestare la competenza internazionale della corte tedesca.

Tale condotta determina l'insorgere della competenza dell'Amtsgericht Flensburg, sebbene sulla base delle disposizioni generali di cui all'art. 3 lett. a) e b) la giurisdizione sarebbe spettata alle corti danesi. Nella motivazione, la corte chiarisce che non è necessaria la verifica dell'esistenza di una eventuale residenza abituale della creditrice presso la madre, risultando la sua competenza ai sensi dell'art. 5, applicabile in seguito alla comparizione della parte, costituitasi senza eccepire l'incompetenza.

Anche l'Oberlandesgericht Stuttgart ${ }^{30}$ si ritiene competente a statuire sulla modifica del mantenimento a favore di due minori residenti abitualmente in Turchia. In precedenza, lo Jugendamt aveva posto a carico del padre, debitore alimentare, il mantenimento dei propri figli minorenni residenti, al pari dello stesso, in Germania. Un anno dopo il trasferimento dei minori in Turchia, il padre adisce l'Amtsgericht Wangen al fine di ottenere la riduzione del mantenimento. I creditori, costituitisi in giudizio, contestano la fondatezza delle pretese attoree senza eccepire l'incompetenza del giudice tedesco.

La decisione di primo grado viene appellata dai creditori ed il giudice di appello, senza che sia sollevata questione sul punto - peraltro non passibile di contestazione in secondo grado- conferma la propria competenza internazionale ai sensi dell'art. 5. La mancata eccezione di incompetenza dei creditori in primo grado ha determinato la competenza internazionale del giudice tedesco, che sarebbe invece spettata alle corti turche.

Dalla sentenza non è dato desumere che i creditori abbiano ritenuto che il giudice tedesco fosse competente ai sensi dell'art. 8 e che non abbiano sollevato eccezione di incompetenza per questo motivo (vedi supra par. II). Appare invece verosimile che i creditori abbiano intenzionalmente prorogato la competenza del giudice tedesco, ritenendo per loro più vantaggioso che la decisione sulla modifica del mantenimento venisse adottata dalle autorità tedesche, anziché da quelle turche.

Le sentenze in esame offrono lo spunto per alcune considerazioni sul peculiare funzionamento dell'art. 5. La proroga tacita attribuisce la competenza al giudice che ne è privo secondo le regole generali, ed opera frequentemente quando convenuto è il creditore.

\footnotetext{
${ }^{28}$ M. ANDRAE, «Kapitel II: Zuständigkeit Art 5 EG-UntVO», in Europäisches Zivilprozess cit., p. 551-555.

${ }^{29}$ AG Flensburg 24 luglio 2014, in I. Ludwig, Juris PraxisKommentar BGB, a cura di M. Herberger, M. Martinek, H. Rüssmann, S. Weth, M. WÜRdingER, Saarbrücken, Juris 2020, ove l'autore critica la scelta della legge applicabile in quanto, dal punto di vista tedesco (e di qualsiasi Stato membro), il protocollo si applica anche alla Danimarca.

${ }^{30}$ OLG Stuttgart 17 gennaio 2014, in Zeitschrift für das gesamte Familienrecht, 2014, p. 850-852.
} 
L'art. 5 deroga al principio del favor creditoris che caratterizza i criteri di giurisdizione degli articoli precedenti, in particolare laddove non prevede la possibilità di indagare sulla corrispondenza tra comparizione senza contestazione e consapevole accettazione della giurisdizione, con effettiva conoscenza delle conseguenze che ne derivano. ${ }^{31}$ Tuttavia, dall'analisi della giurisprudenza, emerge che la disposizione dell'art. 5 può anche favorire il creditore, garantendogli l'accesso ad un ulteriore foro rispetto a quelli già a sua disposizione. Come dimostra il caso deciso dall'Oberlandesgericht Stuttgart, in cui il creditore alimentare era residente in Turchia, non sempre il foro più vantaggioso coincide con quello della residenza abituale del creditore.

\section{La competenza sussidiaria ed il forum necessitatis}

8. Il Reg. n. 4/2009 disciplina la giurisdizione anche nei casi in cui il convenuto o entrambe le parti risiedano abitualmente in un Paese terzo. Gli artt. 6 e 7, ultimi criteri di giurisdizione menzionati, garantiscono infatti "un'ancora di salvezza" per ottenere in uno Stato membro una decisione in materia alimentare. In questo modo, il regolamento deroga anche ai criteri di giurisdizione di fonte internazionale, in particolare quelli posti dalla Convenzione dell'Aia del 2007.

L'art. 6 conferisce competenza a titolo sussidiario alle corti dello Stato membro di cittadinanza comune delle parti, nel caso in cui questa non possa essere determinata né ai sensi degli artt. 3,4 e 5 del regolamento, né ai sensi della Convenzione di Lugano. ${ }^{32}$ Tale criterio quindi, non solo consente di attribuire la competenza ad un giudice dell'Unione europea, ma favorisce il recupero del credito alimentare nelle ipotesi in cui le parti abbiano la cittadinanza comune di uno Stato membro ma risiedano al di fuori dell'Unione europea. ${ }^{33}$

In un caso deciso dall'Oberlandesgericht Koblenz ${ }^{34}$ il debitore chiede la modifica del mantenimento posto a suo carico. Egli risiede abitualmente in Germania, mentre il creditore - suo figlio - in Perù. In primo grado le autorità tedesche dichiarano il difetto di giurisdizione. La sentenza viene appellata dal debitore ma l'Oberlandesgericht Koblenz conferma il difetto di giurisdizione delle autorità tedesche in favore di quelle peruviane, ove il creditore risiede abitualmente.

Il giudice di appello, pur riconoscendo che il Reg. n. 4/2009 trova applicazione anche qualora il convenuto non risieda in uno Stato membro, esclude la propria competenza internazionale anche sulla base dell'art. 6. Infatti, nonostante un indizio che forse entrambe le parti siano cittadine tedesche, nessuna di esse ha fornito sufficienti prove sul punto, impedendo l'operatività del criterio di collegamento della cittadinanza comune.

Considerata la peculiarità del criterio di giurisdizione e del caso in esame, forse il giudice sarebbe potuto giungere ad una differente soluzione. L'autorità tedesca, infatti, pur dichiarando che la competenza internazionale sarebbe potuta risultare dall'art. 6 sulla base di elementi che abbiano fatto presumere l'esistenza di una cittadinanza comune (che in sentenza non vengono però riportati) ${ }^{35}$, si è poi limitata a dichiarare non sufficientemente provata la comune cittadinanza delle parti. ${ }^{36}$ Data la presenza agli atti di un principio di prova in tale senso, il giudice avrebbe forse potuto compiere ulteriori verifiche per accertare tale circostanza e quindi fondare la sua competenza sulla base dell'art. 6. L'applicazione delle norme sulla giurisdizione, come di qualsiasi altra norma giuridica, rappresenta infatti un onere del giudice, al quale lo stesso non può sottrarsi.

\footnotetext{
${ }^{31}$ F. PESCE, Le obbligazioni alimentari cit., p. 143-144.

${ }^{32}$ Convenzione concernente la competenza giurisdizionale, il riconoscimento e l'esecuzione delle decisioni in materia civile e commerciale, conclusa a Lugano il 30 ottobre 2007, è stata firmata dalla Confederazione Svizzera, dalla Comunità europea, dal Regno di Danimarca, dal Regno di Norvegia e dalla Repubblica d'Islanda. Essa operava parallelamente al Reg. $n$. 44/2001. Per gli Stati membri dell'Unione europea, la Convenzione di Lugano del 2007 è entrata in vigore il $1^{\circ}$ gennaio 2010 , in G.U.U.E. n. 147 del 10.6.2009.

${ }^{33}$ M. Castellaneta, A. LeandRo, «Il regolamento CE n. 4/2009» cit., p. 1073-1074.

${ }^{34}$ OLG Koblenz 18 giugno 2014, in Zeitschrift für das gesamte Familienrecht, 2015, p. 268-269.

${ }^{35}$ Dalla sentenza (par. 1) emerge solamente che il creditore è figlio del debitore e vive dal 2013 con la madre, divorziata dal debitore, in Perù.

${ }^{36}$ Cfr. par. 8 della sentenza.
} 
9. L'art. 7 consente ad uno Stato membro di conoscere della controversia quando questa non possa essere instaurata davanti al giudice di uno Stato terzo.

Nessun'altra autorità di uno Stato membro deve però essere competente ai sensi degli artt. 3,4,5 e 6. Per potersi applicare l'art. 7 è necessario che sussista un collegamento tra la controversia e lo Stato membro adito, che può essere integrato anche dalla semplice presenza fisica nel Paese in questione. ${ }^{37}$

Il forum necessitatis rappresenta una novità nel campo dei regolamenti dell'Unione europea $\mathrm{e}$ si inserisce nel sistema di cooperazione in materia civile. In quanto norma di chiusura delle disposizioni attributive della giurisdizione, l'art. 7 scongiura il pericolo di un non liquet, e cioè che la controversia non possa essere risolta a causa della assenza di un giudice competente sulla base dei criteri generali. ${ }^{38}$ Difficoltà applicative si riscontrano nella prova dei presupposti per la sua operatività, i quali ne richiedono e determinano un impiego assai prudente.

Considerata la rara applicazione del forum necessitatis, merita di essere citata la sentenza del Bundesgerichtshof del $2015^{39}$ nella quale tale giudice, dopo aver analizzato le diverse strade percorribili dal debitore di alimenti residente in Germania per ottenere la modifica del mantenimento di un creditore residente negli Stati Uniti d'America, applica l'art. 7 mostrandone la concreta operatività.

Nel caso di specie, il Bundesgerichtshof rigetta le conclusioni cui era pervenuto il giudice di appello, secondo cui una decisione sul mantenimento adottata dal giudice tedesco ${ }^{40}$ deve essere modificata dalle autorità giudiziarie americane, in quanto foro di residenza del creditore.

Secondo il giudice di legittimità il debitore avrebbe difficoltà ad ottenere tutela di fronte alle autorità statunitensi. Stante l'assenza di convenzioni in materia alimentare, la giurisdizione sul punto è determinata dalle norme interne ${ }^{41}$ Il sistema processuale americano è però retto dal principio della "continuing exclusive jurisdiction" secondo cui una decisione non può essere modificata in un Paese diverso rispetto a quello in cui sia stata presa. Considerato che la prima decisione era stata resa dal giudice tedesco, il Bundesgerichtshof ritiene che le autorità americane avrebbero declinato la loro competenza.

L'aspetto di maggiore interesse si rinviene però nella seconda parte della pronuncia, laddove il Bundesgerichtshof radica la competenza del giudice tedesco sull'art. 7 nell'ipotesi in cui, come verosimilmente nel caso di specie, il debitore non possa ottenere tutela di fronte alle corti americane. Secondo il giudice di legittimità questa disposizione, fungendo da clausola di salvaguardia, evita che conflitti internazionali negativi di competenza determinino un diniego di giustizia.

La stessa ratio è altresì rinvenibile nell'art. 8 comma 2 lett. c) il quale esclude la perpetuatio fori (comma 1) nel caso in cui il giudice di residenza del creditore, competente in quanto Stato di origine, e «contraente della convenzione dell'Aia del 2007 non possa o rifiuti di esercitare la competenza a modificare la decisione o a emetterne una nuova».

\footnotetext{
${ }^{37}$ F. C. Villata, Obblighi alimentari cit., p. 753-754; F. PoCaR, «La disciplina comunitaria» cit., p. 13-14.

${ }^{38} \mathrm{G}$. BiagionI, «Alcuni caratteri generali del forum necessitatis nello spazio giudiziario europeo», in Cuadernos de Derecho Transnacional,2012, p. 26-29.

${ }^{39}$ BGH 14 ottobre 2015, in Zeitschrift für das gesamte Familienrecht, 2016, p. 115-116.

${ }^{40} \mathrm{Il}$ creditore, residente negli Stati Uniti d'America, presentava di fronte al giudice tedesco di primo grado istanza volta ad ottenere la dichiarazione giudiziale di paternità; in tale occasione, il creditore chiedeva la condanna del debitore al pagamento degli alimenti a partire dal momento della sua nascita.

${ }^{41}$ Sebbene sia stato uno dei primi Paesi a firmare la Convenzione sull'esazione internazionale di prestazioni alimentari nei confronti di figli e altri membri della famiglia, all'epoca della sentenza in esame gli Stati Uniti non avevano ancora proceduto alla sua ratifica. Solo in data 7 settembre 2016 è stato depositato lo strumento di ratifica, divenendo gli Stati Uniti il trentatreesimo Paese ad applicare tale convenzione, entrata in vigore il $1^{\circ}$ gennaio 2017. Essa è stata conclusa il 23 novembre 2007 ed approvata a nome dell'Unione europea, con Decisione del Consiglio 2011/432/UE del 9 giugno 2011, in G.U.U.E. n. L 192/39 del 22 luglio 2011. La Convenzione dell'Aia del 2007 contiene solamente norme indirette e negative di giurisdizione. Con riferimento al primo aspetto l'art. 20, collocato nel capo dedicato al riconoscimento ed all'esecuzione, consente il riconoscimento di una decisione solamente nell'ipotesi in cui uno Stato contraente abbia rispettato parte dei requisiti dallo stesso prescritti. L'art. 18, contiene invece una norma di competenza negativa secondo la quale, fintantoché il creditore continui a risiedere abitualmente nello Stato contraente in cui sia stata emessa una decisione in materia di obbligazioni alimentari, essa non può essere oggetto di modifica o di nuova statuizione da parte del debitore in uno Stato diverso. Nel caso di specie, tuttavia, il creditore non ha mai risieduto in Germania e per tale ragione l'art. 18 non può trovare applicazione.
} 
Secondo il giudice tedesco non è infatti ipotizzabile che un regolamento dell'Unione europea non garantisca al debitore, che sia abitualmente residente in uno Stato membro, un foro ove ottenere la modifica di una decisione relativa al mantenimento di un creditore residente all'estero.

La pronuncia del Bundesgerichtshof offre lo spunto per qualche riflessione sull' operatività del forum necessitatis. La realizzazione delle pretese alimentari è garantita dall'art. 7 nell'ipotesi in cui si possano verificare conflitti negativi di giurisdizione. L'applicazione di questo articolo è infatti subordinata al fatto che non sia possibile ottenere tutela nello Stato terzo con cui la controversia presenti un collegamento. Solo in ipotesi di limitazioni in fatto o in diritto l'art. 7 trova applicazione e conferisce competenza allo Stato membro con il quale è rinvenibile un legame. ${ }^{42}$

\section{La giurisdizione per gli enti pubblici}

10. Le corti tedesche hanno più volte affrontato il problema di quale sia il foro competente per le domande promosse dagli enti pubblici.

Gli enti pubblici conferiscono prestazioni sociali e ricoprono un ruolo centrale nel fornire assistenza ai creditori, in particolare laddove le pretese di questi ultimi siano rimaste insoddisfatte. Per tale ragione, anche essi necessitano di strumenti che consentano loro di ottenere il rimborso delle prestazioni erogate in luogo del debitore principale. ${ }^{43}$ Tale possibilità è prevista dal regolamento sulle obbligazioni alimentari, il quale facilita il recupero effettivo dei crediti alimentari transfrontalieri anche da parte degli enti pubblici.

Ai sensi dell'art. 1 Reg. n. 4/2009 rientrano nell'ambito di applicazione del regolamento anche quei procedimenti nei quali gli enti pubblici agiscono nei confronti dei debitori in rappresentanza dell'avente diritto o in regresso per prestazioni adempiute a quest'ultimo in luogo del mantenimento. ${ }^{44} \mathrm{Si}$ pone però il problema dell'individuazione del foro presso il quale l'ente pubblico può instaurare la causa nei confronti del debitore, in particolare quando agisce in regresso.

Nel primo caso, infatti, quando l'ente pubblico agisce in nome e per conto del creditore, gli effetti giuridici sono imputabili a quest'ultimo.

Il creditore è parte in senso sostanziale mentre l'ente pubblico in senso formale, quindi l'instaurazione della causa nel foro della residenza abituale del creditore non pone particolari problemi teorici. ${ }^{45}$

Il discorso è diverso quando l'ente pubblico, surrogatosi nei diritti del creditore, agisce in regresso nei confronti del debitore. In questo caso l'ente pubblico è parte sia in senso formale sia in senso sostanziale. In questa ipotesi ci si chiede se l'ente pubblico, oltre che nel foro del debitore, possa agire anche nel proprio foro (ad esempio nel luogo in cui ha sede l'autorità pubblica), in qualità di "creditore". A tale possibilità si oppone però il dato letterale dell'art. 2 n. 10 Reg. n. 4/2009, il quale dichiara esplicitamente che "creditore" è solo una persona fisica.

Se dunque l'ente pubblico non può instaurare la causa di regresso nel proprio foro, questi dovrebbe poter agire nel foro della residenza abituale del creditore originario. Il fondamento giuridico di questa azione pone altrettanti problemi, in quanto il creditore, a seguito della surroga dell'ente pubblico nei suoi diritti, perde il ruolo di parte. A sostegno di questa possibilità si è ritenuto che, se l'obiettivo dell'azione di regresso consiste nel recupero degli alimenti, la relazione giuridica fra creditore ed ente pubblico non può incidere sull'applicazione dei criteri di giurisdizione. Considerato che l'ente pubblico si surroga nel diritto vantato dal creditore originario, sostituendosi a quest'ultimo, è sostenibile che il primo, dopo il pagamento della prestazione sociale, possa instaurare la causa nel foro del titolare originario. ${ }^{46}$

\footnotetext{
${ }^{42}$ G. BIAGIONI, «Alcuni caratteri generali del forum necessitatis» cit., p. 32-33.

${ }^{43}$ P. Beaumont, «International Family Law in Europe - the Maintenance Project, the Hague Conference and the EC: A Triumph of Reverse Subsidiarity», in The Rabel Journ of Comp. and Int. Priv. Law, 2009, p. 539.

${ }^{44}$ M. ANDRAE, «Kapitel I: Anwendungsbereich und Begriffsbestimmungen Art 1 EG-UntVO», in Europäisches Zivilprozess cit., p. 488-489.

${ }^{45}$ V. LIPP, EG-UntVO Art. 1 Anwendungsbereich, in Münchener Kommentar zum FamFG, a cura di T. RAuscher, München, C.H. Beck 2019.

${ }^{46}$ M. ANDRAE, «Kapitel I: Anwendungsbereich und Begriffsbestimmungen», in Europäisches Zivilprozess cit., p. 488-489.
} 
11. Tale possibilità è stata però negata con riferimento alla Convenzione di Bruxelles del 1968 e, successivamente, al Reg. n. 44/2001. Con la sentenza Blijdenstein, ${ }^{47}$ la Corte di giustizia dell'Unione europea ha chiarito infatti che, pur rientrando l'azione di regresso di un ente pubblico nei confronti di un debitore nel campo di applicazione della convenzione suddetta (art. 1), l'ente pubblico non può adire il foro di cui all'art. 5 n. 2. Secondo tale disposizione, ripresa testualmente dal Reg. n. 44/2001, le persone che sono domiciliate nel territorio di uno Stato membro possono essere convenute «davanti al giudice del luogo in cui il creditore di alimenti ha il domicilio o la residenza abituale».

Questa interpretazione è fondata sulla considerazione che un ente pubblico che agisce in via di regresso nei confronti del debitore di alimenti non si trova in una posizione subordinata equiparabile a quella del creditore alimentare, tale da giustificare l'applicazione dei criteri speciali di giurisdizione e privare il debitore della tutela del foro del proprio domicilio ${ }^{48}$ (art. 2 Convenzione di Bruxelles del 1968). ${ }^{49}$

Successivamente all'entrata in vigore del Reg. n. 4/2009, è controverso se tale limitazione si applichi anche a quest'ultimo strumento. ${ }^{50}$

Si sono così delineati due diversi indirizzi. Da un lato, in virtù del noto rapporto di continuità tra gli strumenti normativi in questo campo, si ritiene la citata giurisprudenza applicabile anche al regolamento, per cui un'azione di regresso di un ente pubblico nei confronti di un debitore può solo essere instaurata nel foro di quest'ultimo e non in quello dell'art. 3 lett. b). Questo orientamento si basa sul considerando $14^{51}$ e sull'art. 64 del regolamento, i quali estendono il concetto di creditore agli enti pubblici (ex art. 2 n. 10) esclusivamente nelle ipotesi di procedimenti di riconoscimento ed esecuzione e lo escludono invece nelle fasi precedenti del giudizio. Inoltre, il silenzio del regolamento sul punto sembra confermare quanto suesposto.

Dall'altro, l'indirizzo opposto ritiene che escludere gli enti pubblici dalla possibilità di usufruire del foro della residenza abituale del creditore contrasti con gli obiettivi del Reg. n. 4/200952 (cons. n. 45).$^{53} \mathrm{~L}$ 'apertura all'ente pubblico del foro del creditore consente infatti, da una parte, di avere accesso ad un foro nell'Unione europea qualora il debitore, diversamente dal creditore, risieda in uno Stato terzo; dall'altra, favorisce la sempre auspicata corrispondenza tra forum e ius. ${ }^{54} \mathrm{Il}$ punto, come vedremo tra breve, è stato risolto dalla Corte di giustizia dell'Unione europea.

12. Sul presupposto dell'impossibilità per gli enti pubblici di usufruire del foro del creditore nelle azioni di regresso, la prassi tedesca ha cercato altre strade per consentire agli enti pubblici di recuperare le somme di denaro davanti al giudice tedesco, trovando una soluzione sulla base di alcune disposizioni di legge vigenti, ${ }^{55}$ mediante un meccanismo complesso basato su una doppia cessione del titolo.

Questo consiste nella cessione da parte dell'ente pubblico al creditore originario dei crediti alimentari nel cui diritto il primo si sia surrogato (a seguito del pagamento dell'obbligazione del debi-

\footnotetext{
${ }^{47}$ Corte di giustizia 15 gennaio 2004, Freistaat Bayern c. Blijdenstein, 433/2001.

${ }^{48}$ Ivi, punto 29,30 e 31 .

${ }^{49}$ R. Hausmann, «Unterhaltssachen», in Internationales und Europäisches Familienrecht, a cura di R. Hausmann, München, C.H. Beck 2018; F. Pesce, Le obbligazioni alimentari cit. p. 90-91; F. C. Villata, «Obblighi alimentari» cit., p. 743-744.

${ }^{50}$ P. M. Reuss, «Art. 3 lit. b EuUntVO: Internationale Zuständigkeit für grenzüberschreitenden Behördenregress bei Elternunterhalt», in Zeitschrift für das gesamte Familienrecht, 2019, p.1341-1342.

${ }^{51}$ Il quale recita: «E opportuno che il presente regolamento preveda che il termine "creditore" comprende, ai fini di una domanda di riconoscimento e di esecuzione di una decisione in materia di obbligazioni alimentari, gli enti pubblici che hanno il diritto di agire per conto di una persona cui siano dovuti alimenti o di chiedere il rimborso di prestazioni erogate al creditore in luogo degli alimenti. Se l'ente pubblico agisce in tal veste, dovrebbe aver diritto agli stessi servizi e allo stesso patrocinio a spese dello Stato al pari del creditore».

52 W. WuRMnest, «EU-UnterhaltsVO Art. 3 Allgemeine Bestimmungen», in Grosskommentar zum Zivilrecht, a cura di B. Gsell, W. Krüger, S. Lorenz, C. Reymann, München, C.H. Beck 2019.

${ }^{53}$ Questi considera obiettivi del presente regolamento l'istituzione «di una serie di misure che permettano di garantire il recupero effettivo dei crediti alimentari in situazioni transfrontaliere e agevolare in tal modo la libera circolazione delle persone all'interno dell'Unione europea».

${ }^{54}$ V. LiPP, «Vorbemerkung zu Art. 3», in Münchener Kommentar cit., Rn. 29-34.

${ }^{55}$ Ex multis, art. 33 comma 4 Sozialgesetzbuch (SGB) II e art. 94 comma 5 Sozialgesetzbuch (SGB) XII.
} 
tore) ${ }^{56}$ così da permettere al creditore originario di avviare il procedimento nel proprio foro. ${ }^{57}$ In tale procedura, quest'ultimo rilascia una procura a favore dell'ente pubblico, che conduce autonomamente il processo o si serve di un avvocato. ${ }^{58}$

Nel 2013, in un caso relativo ad una coppia turca separata e residente in Germania, l'Amtsgericht Stuttgart ${ }^{59}$ prende le distanze dalla sentenza Blijdenstein e ritiene che l'ente pubblico possa instaurare la causa nel foro della residenza abituale del creditore originario.

Prima del trasferimento del debitore in Turchia, quest'ultimo veniva condannato al mantenimento della moglie residente, come questi, in Germania.

Poiché tale obbligazione non veniva adempiuta, la creditrice aveva ricevuto un assegno sociale dallo Stato tedesco.

Il Landratsamt B., surrogatosi nei diritti della creditrice verso il debitore in virtù dell'adempimento all'obbligazione originaria in luogo di quest'ultimo, cedeva alla moglie i crediti nei quali si era surrogato, così da consentirle di farli valere in nome proprio. Questa instaurava perciò la causa di fronte all'autorità giudiziaria tedesca, foro della propria residenza abituale.

Secondo l'Amtsgericht Stuttgart, poiché il Reg. n. 4/2009 pone su un piano di parità i criteri di giurisdizione, esso consente al creditore una scelta del foro che perdura anche nell'ipotesi in cui l'ente pubblico, surrogatosi nei diritti del creditore originario, ritrasferisca a quest'ultimo il credito alimentare. $\grave{E}$ al foro del creditore (originario) persona fisica che l'art. 2 comma $1 \mathrm{n}$. 10) si riferisce, e non a colui che si sia surrogato nei diritti di questi. A sostegno di questa posizione, l'Amtsgericht Stuttgart sottolinea che nell'ipotesi in cui venga precluso all'ente pubblico di utilizzare il foro di cui all'art. 3 lett. b), può diventare necessario adire due autorità giudiziarie distinte. Infatti, i crediti non trasferiti si devono far valere nel luogo della residenza abituale del creditore, mentre i crediti ceduti all'ente pubblico solamente in quella del debitore, determinando una frammentarietà contraria ai principi ispiratori dei regolamenti dell'Unione europea.

Nella sentenza dell'Amtsgericht Stuttgart si discute della possibilità per l'ente pubblico di agire in regresso verso il debitore di alimenti nel foro della residenza abituale del creditore ex art. 3 lett. b). Il giudice tedesco ritiene che le limitazioni interpretative in vigenza degli strumenti anteriori al regolamento non siano più applicabili a quest'ultimo e che consentire all'ente pubblico di accedere al foro del creditore meglio si concili con lo spirito e con gli obiettivi del regolamento. In questo modo, si esclude che il meccanismo di trasferimento del credito adottato nella prassi continui ad essere messo in atto nelle ipotesi in cui l'ente pubblico, surrogatosi nei diritti del creditore, voglia agire in regresso nei confronti del debitore. Infatti, avendo l'ente pubblico la facoltà di instaurare la causa nel foro di cui all'art. 3 lett. b) una tale cessione diviene priva di scopo. ${ }^{60}$

13. L'assenza di un orientamento uniforme, tanto nella giurisprudenza quanto nella dottrina, porta in tempi recentissimi il Bundesgerichtshof ${ }^{61}$ a sottoporre alla Corte di giustizia dell'Unione europea una questione pregiudiziale sull'interpretazione dell'art. 3 lett. b) Reg. n. 4/2009.

La vicenda concerne un ente pubblico tedesco che agisce in regresso in Germania nei confronti di un debitore alimentare residente in Austria, al fine di recuperare i crediti alimentari in cui lo stesso si è

${ }^{56}$ L'art. 33 Sozialgesetzbuch (SGB) II è intitolato "Übergang von Ansprüchen" (trasferimento dei crediti); il comma 1 prevede che il titolare di un diritto a ricevere una prestazione sociale trasferisca questo diritto all'ente pubblico che gli abbia fornito la stessa in luogo della persona obbligata.

${ }^{57}$ Ai sensi dell'art. 33 comma 4 Sozialgesetzbuch (SGB) II, su accordo del beneficiario delle prestazioni (il creditore originario), gli enti possono cedere a quest'ultimo, ai fini di un procedimento giudiziario, il diritto loro trasferito per poi farsi nuovamente cedere il credito così rivendicato.

${ }^{58}$ P. MANKowsKI, «Die internationale Zuständigkeit nach Art. 3 EuUnterhVO und der Regress öffentlicher Einrichtungen», in Praxis des Internationalen Privat- und Verfahrensrechts, 2014, p. 254; D. MARTINY, «Jurisdiction, Recognition and Enforcement in Cases of Reimbursement Claims by Public Bodies», in The Recovery of Maintenance in the EU and Worldwide, a cura di P. Beaumont, B. Hess, L. Walker, S. Spancken, Oxford and Portland, Oregon, Hart Publishing 2014, p. 489-490.

${ }^{59}$ AG Stuttgart 4 settembre 2013, in Zeitschrift für das gesamte Familienrecht, 2014, p. 786-787.

${ }^{60}$ P. MANKOWSKI, «Die internationale Zuständigkeit» cit., p. 249.

${ }^{61}$ BGH 5 giugno 2019, in Zeitschrift für das gesamte Familienrecht, 2019, p. 1340-1345. 
surrogato ex art. 94 comma 1 Sozialgesetzbuch (SGB) XII. ${ }^{62}$ La creditrice originaria, madre del debitore e titolare di assistenza sociale, risiede in un ospizio in Germania. Il debitore contesta la competenza del giudice tedesco.

In primo grado il giudice tedesco nega la sua giurisdizione non potendosi considerare l'ente pubblico creditore ai sensi all'art. 3 lett. b). L'Oberlandesgericht Köln, al contrario, ritenendo applicabili i criteri di cui all'art. 3 lett. a) e b) anche all'ente pubblico surrogatosi nel credito alimentare, annulla la decisione impugnata e rinvia la causa al giudice di primo grado per una statuizione sul merito.

Contro la decisione del giudice di appello, il debitore ricorre al Bundesgerichtshof, il quale ritiene necessario un rinvio alla Corte di giustizia. Nel corso del giudizio vengono sostenuto opposte tesi. Da una parte vi è chi sostiene, ${ }^{63}$ conformemente alla sentenza Blijdenstein, che un ente pubblico che agisca in regresso nei confronti di un debitore non si trovi in una posizione di inferiorità nei confronti di quest'ultimo e non possa quindi usufruire del foro della residenza abituale del creditore. L'unico foro a disposizione dell'ente per un'azione di regresso è pertanto quello della residenza abituale del convenuto, di cui all'art. 3 lett. a).

Dall'altra parte invece, ${ }^{64}$ si sostiene che nel Reg. n. 4/2009 la competenza del giudice della residenza abituale del creditore non possa più considerarsi un'eccezione, in quanto il regolamento prevede criteri di competenza alternativi di pari rango. Il giudice del rinvio appoggia questo secondo indirizzo, basandosi sia su un'interpretazione estensiva dell'art. 64 del regolamento, sia sugli obiettivi dallo stesso perseguiti.

La Corte di giustizia, con sentenza WV c. Landkreis Harburg del 17 settembre 2020, ${ }^{65}$ seguendo l'opinione espressa dal suo avvocato generale e dalla Commissione, chiarisce che l'ente pubblico che agisce in regresso nei confronti del debitore è legittimato ad avvalersi anche del foro della residenza abituale del creditore originario. I principi giurisprudenziali sviluppatisi nel solco degli strumenti precedenti non possono essere trasposti meccanicamente nel Reg. n. 4/2009, in quanto è necessario che nell'interpretare le disposizioni sulla competenza internazionale di quest'ultimo vengano compiute considerazioni di carattere sia sistematico sia teleologico. ${ }^{66}$

La struttura della Convenzione di Bruxelles del 1968 è molto differente da quella del Reg. n. 4/2009. In quest'ultimo non vi è né un criterio di giurisdizione generale (quale quello del domicilio del convenuto alla base della Convenzione di Bruxelles), né una norma speciale che deroghi questa regola, come quella prevista all'art. 5 n. 2 per le obbligazioni alimentari. Inoltre, l'art. 3 Reg. n. 4/2009 non specifica se legittimato all'azione nei fori di cui alla lett. a) e b) debba essere solo il creditore.

La Corte conclude pertanto che l'ente pubblico può agire in regresso nel foro della residenza abituale del creditore. Ciò facilita inoltre la riscossione del credito alimentare transfrontaliero, garantendo all'ente la disponibilità di un foro all'interno dell'Unione europea nell'ipotesi in cui il debitore sia residente in uno Stato terzo. La Corte osserva che tale interpretazione non compromette neppure il principio della prevedibilità delle norme sulla competenza, in quanto il debitore è già a conoscenza del fatto di poter essere citato di fronte ai giudici del luogo di residenza abituale del creditore.

Da ultimo, aprire il foro di cui all'art. 3 lett. b) all'ente, il quale spesso risiede nel medesimo luogo del creditore, garantisce la corrispondenza tra forum e ius, conformemente al Protocollo dell'Aia del 2007.

La pronuncia risolve un conflitto interpretativo che, come accennato, ha generato decisioni contrastanti ed ostacolato l'applicazione uniforme del regolamento.

\footnotetext{
${ }^{62}$ Il quale, nella prima parte, recita: «Se, per il periodo di erogazione delle prestazioni, la persona avente diritto alla prestazione vanta, in base al diritto civile, crediti alimentari, questi si trasferiscono in capo al soggetto responsabile dell'assistenza sociale, sino a concorrenza delle spese sostenute unitamente al diritto di accesso previsto dalla normativa in materia di alimenti».

${ }^{63}$ W. Hau, «EuUntVO», in FamFG, a cura di H. Prütring, T. Helms, Köln, Dr. Otto Schmidt, 2020, Rn. 40; R. HausmanN, «Unterhaltssachen» cit.; N. ConTı, Grenzüberschreitende Durchsetzung von Unterhaltsansprüchen in Europa, Köln, Dr. Otto Schmidt 2011, p. 192 ss.; D.-C. BitTMAnN, «EuUntVO», in Zivilrecht unter europäischem Einfluss, a cura di M. GeBauER, T. WiedmanN, Stuttgart, C.H. Beck 2010, Rn. 33.

${ }^{64}$ V. LiPP, «EG-UntVO Art. 1 Anwendungsbereich», in Münchener Kommentar cit., Rn. 55 ss.; M. AndraE, «Kapitel I: Anwendungsbereich und Begriffsbestimmungen Art 1 EG-UntVO», in Europäisches Zivilprozess cit., Rn. 45 ss.

${ }^{65}$ Corte di giustizia 17 settembre 2020, WV c. Landkreis Harburg, 540/19.

${ }^{66} \mathrm{Si}$ fa riferimento ai punti 24 e 25 della sentenza Corte di giustizia 18 dicembre 2014, Sanders e Huber, 400/13 e 408/13.
} 
È dubbio, tuttavia, che una tale apertura, seppur da molti auspicata, ${ }^{67}$ rappresenti una soluzione davvero equa. Nonostante la Corte abbia fatto presente che il criterio di competenza di cui all'art. 3 debba essere interpretato «alla luce delle sue finalità, del suo tenore letterale, nonché del sistema all'interno del quale esso si colloca ${ }^{68}$ ci si chiede se sia prudente abbandonare la valutazione sull'equilibrio del rapporto tra le parti che, nel passato, ha escluso l'estensione all'ente pubblico del foro del creditore. Il debitore di alimenti, sempre persona fisica, ed un ente pubblico non si trovano infatti mai in una posizione di parità economica. Pare dunque che debba essere valutata con prudenza l'apertura del foro del creditore - espressione di un favor processuale per una parte considerata "debole" - al soggetto più forte economicamente, in questo caso l'ente pubblico.

Per quanto si riconosca che l'ente pubblico sia obbligato a versare somme di denaro in luogo degli alimenti verso un numero sempre maggiore di soggetti e che sia necessario facilitarne il recupero, si deve anche tenere presente che pure il debitore inadempiente può essere una persona in difficoltà economiche. Nella causa di regresso l'equilibrio tra le parti verrebbe così alterato, costringendo il debitore a difendersi in un foro che, per quanto prevedibile, è comunque lontano da chi è «nella posizione migliore per valutare le risorse di quest'ultimo». ${ }^{69}$

\section{Il Protocollo dell'Aia del 2007}

14. Al tema della legge applicabile, il Reg. n. 4/2009 dedica esclusivamente l'art. 15 il quale, tramite un rinvio formale, dispone che questa «è determinata secondo il Protocollo dell'Aia del 23 novembre 2007 relativo alla legge applicabile alle obbligazioni alimentari [...] negli Stati membri vincolati da tale strumento»». ${ }^{70}$

La disciplina della legge applicabile viene quindi collocata al di fuori sia del regolamento, sia della Convenzione dell'Aia del 2007, alla cui adesione rimane svincolato. ${ }^{71}$ Questa soluzione di compromesso, giunta in un momento avanzato del processo di negoziazione del regolamento, nasce con l'obiettivo di consentire un'ampia adesione allo strumento europeo, nonché allo scopo di incentivare Paesi (quali ad esempio il Regno Unito) ad applicarlo. ${ }^{72}$ Infatti, tutti gli "Stati membri”, ad eccezione del Regno di Danimarca e del Regno Unito, sono vincolati al protocollo. ${ }^{73}$

Quest'ultimo, inoltre, grazie all'adesione dell'Unione europea, ${ }^{74}$ è considerato una fonte di diritto secondario, soggetta quindi all'interpretazione della Corte di giustizia, la quale può risultare da un rinvio pregiudiziale di interpretazione, come disciplinato dall'art. 267 TFUE. ${ }^{75}$

Anche il protocollo, come già il regolamento, armonizza unicamente le norme di diritto internazionale privato uniformi in materia di obbligazioni alimentari «derivanti da rapporti di famiglia, di parentela, di matrimonio o di affinità comprese le obbligazioni alimentari nei confronti dei figli a prescindere dallo stato civile dei genitori». Il protocollo non determina dunque la legge applicabile ai rapporti di famiglia da cui derivano le obbligazioni alimentari, in quanto tale aspetto è disciplinato dalla legge nazionale di ciascuno Stato. ${ }^{76}$

${ }^{67}$ Per una visione favorevole all'apertura del foro del creditore all'ente pubblico v. P. MANKowsKI, «Die internationale Zuständigkeit» cit., p. 252.

${ }^{68}$ Corte di giustizia 18 dicembre 2014, Sanders e Huber cit., punto 25.

${ }^{69}$ Corte di giustizia 17 settembre 2020, WV c. Landkreis Harburg cit., punto 31.

${ }^{70}$ I. VIARENGO, «Sulla disciplina degli obblighi alimentari» cit., p. 825-826.

${ }^{71}$ N. Contı, V. BisSMEIER, «Das neue Haager Unterhaltsprotokoll von 2007», in FamilienRechtsberater, 2011, p. 62.

${ }^{72}$ M. C. BARUfFI, «In tema di riconoscimento delle decisioni» cit., p. 135.

${ }^{73}$ Perunapanoramica sugli Statiparte del protocollo: https://www.hcch.net/en/instruments/conventions/status-table/?cid=133.

${ }^{74}$ L'Unione europea ha aderito al protocollo sulla base dell'art. $300 \mathrm{CE}$ (oggi art. $218 \mathrm{TFUE}$ ) con effetto per tutti gli "Stati membri" ad eccezione del Regno di Danimarca e del Regno Unito, in G.U.U.E. n. 331 del 16.12.2009. L'Unione europea lo ha poi ratificato in data 8 aprile 2010 ed applicato in via provvisoria dal 18 giugno 2011.

${ }^{75}$ A. Davì, A. ZANoBETTI, «Le obbligazioni alimentari tra parti di un'unione civile e tra conviventi nel diritto internazionale privato», in Rivista trimestrale di diritto e procedura civile, 2017, p. 206-207.

${ }^{76}$ Ciò trova riscontro sia nell'art. 1 comma 2 protocollo, sia nell'art. 22 Reg. n. 4/2009. Invero, ai sensi del primo: «Le decisioni emesse in applicazione del presente protocollo non pregiudicano l'esistenza di uno dei rapporti di cui al paragrafo $1 »$. 
Al pari della Convenzione dell' Aia del $1973,{ }^{77}$ il protocollo ha portata universale ed i suoi effetti sono erga omnes in quanto determina l'applicazione anche di norme appartenenti a Stati non contraenti. ${ }^{78}$ Così, nell'ipotesi in cui un creditore residente in uno Stato che non abbia aderito al protocollo instauri una causa nel foro del debitore, la legge dello Stato di residenza del primo deve comunque trovare applicazione. ${ }^{79}$ Stessa cosa nel caso in cui il creditore sia citato nel proprio foro, situato in un Paese che non ha aderito al protocollo; la legge applicabile alla controversia è comunque quella dello Stato di residenza abituale del creditore.

Ai sensi dell'art. 3 protocollo, le obbligazioni alimentari sono infatti disciplinate dalla legge dello Stato di residenza abituale del creditore. Come per la competenza internazionale, il cambiamento di residenza comporta l'applicazione di una nuova legge (art. 3 comma 2).

Gli artt. 4, 5 e 6 contengono norme cd. speciali che si applicano nei confronti di diversi soggetti del rapporto alimentare. Nello specifico l'art. 4 concerne le obbligazioni alimentari dei genitori nei confronti dei figli (e viceversa) e di persone diverse dai genitori nei confronti di soggetti di età inferiore agli anni ventuno, ad esclusione dei rapporti tra coniugi o ex coniugi. Questi sono regolati dall'art. 5, il quale consente di applicare la legge dello Stato dell'ultima residenza abituale comune (o altra che presenti uno stretto collegamento con il matrimonio) in luogo di quella determinata dall'art. 3 quando una delle parti ne faccia richiesta. ${ }^{80}$

L'art. 6 consente al debitore, fuori dai casi di rapporti di filiazione o di coniugio, di opporsi alla pretesa creditoria, dimostrando l'inesistenza di un'obbligazione alimentare sia ai sensi della legge dello Stato di residenza abituale, sia di cittadinanza comune. Tale disposizione trova applicazione nei rapporti tra cugini o fra suoceri e generi. ${ }^{81}$

Il protocollo ammette anche la scelta della legge applicabile. Questa può avvenire ai fini di un determinato procedimento (art. 7) o, in via generale, per disciplinare il rapporto alimentare tra le parti (art. 8). Nel primo caso, la designazione è finalizzata ad una specifica controversia, è ammissibile anche nei confronti di un minore e deve rispettare determinati requisiti formali. ${ }^{82}$ L'art. 8 , invece, consente alle parti solo di accedere ad una lista predeterminata di leggi e non ammette scelta nel caso in cui essa riguardi minori di anni diciotto o soggetti legalmente incapaci (art. 8 comma 3).

La legge determinata dal protocollo non trova applicazione «soltanto nella misura in cui produca effetti manifestamente contrari all'ordine pubblico del foro» (art. 13).

15. In Germania, le disposizioni del protocollo non sembrano creare particolari problemi applicativi.

Tuttavia, è interessante analizzare la pronuncia della Hanseatisches Oberlandesgericht Bre$m e n^{83}$ nella quale si discute della legge applicabile alla modifica di una decisione sul mantenimento. Il caso è però del tutto particolare in quanto il diritto sul quale si è fondata la decisione originaria è stato determinato in modo errato e, allo stesso tempo, non è avvenuto alcun cambio di residenza da parte del creditore che consenta di applicare una nuova legge.

Anche l'art. 22 stabilisce che: «Il riconoscimento e l'esecuzione di una decisione in materia di obbligazioni alimentari a norma del presente regolamento non implicano in alcun modo il riconoscimento del rapporto di famiglia, di parentela, di matrimonio o di affinità alla base dell'obbligazione alimentare che ha dato luogo alla decisione».

${ }^{77}$ Ai sensi dell'art. 3 della convenzione: «The law designated by this Convention shall apply irrespective of any requirement of reciprocity and whether or not it is the law of a Contracting State».

${ }^{78}$ I. VIARENGO, «Sulla disciplina degli obblighi alimentari» cit., p. 827.

${ }^{79}$ A. Bonomi, «The Hague Protocol of 23 November 2007 on the Law Applicable to Maintenance Obligations», in Yearbook of Priv. Int. Law, 2008, p. 337.

${ }^{80}$ Tale previsione nasce dall'idea secondo cui la legge di residenza abituale del creditore rappresenti una tutela eccessiva qualora essa si trovi ad operare nei confronti di coniugi o ex coniugi. In assenza della disposizione di cui all'art. 5, sarebbe infatti consentito al creditore di determinare unilateralmente la legge applicabile al mantenimento attraverso un repentino cambio di residenza precedente all'instaurazione della causa sugli alimenti.

${ }^{81}$ N. CONTI, V. BISSMEIER, «Das neue Haager Unterhaltsprotokoll» cit., p. 66.

${ }^{82}$ L'art. 7 comma 2 chiarisce che la scelta della legge applicabile deve formare oggetto di un accordo, sottoscritto da entrambe le parti e redatto in forma scritta o registrato su un supporto accessibile in caso di successive consultazioni.

${ }^{83}$ OLG Bremen 17 ottobre 2016, in Zeitschrift für das gesamte Familienrecht, 2017, p. 614-617. 
Il debitore risiede da diversi anni in Polonia mentre il creditore, suo figlio, in Germania. Nel 2016 l'Amtsgericht Bremen, su richiesta del debitore, libera quest'ultimo dall'obbligo di provvedere al mantenimento posto a suo carico dal giudice polacco con la pronuncia di divorzio. Il tribunale tedesco applica la legge tedesca, in quanto legge del luogo di residenza del creditore.

L'Hanseatisches Oberlandesgericht Bremen ritiene invece fondato l'appello del creditore, il quale contesta l'applicazione del diritto tedesco in luogo di quello polacco.

Secondo il giudice di appello, infatti, laddove non si sia verificato alcun cambio di residenza del creditore di alimenti a seguito della decisione originaria, la legge su cui si è basata quest'ultima rimane invariata, indipendentemente dal fatto che il diritto applicabile sia stato correttamente individuato o meno.

Nel caso di specie, infatti, sebbene il minore risiedesse in Germania, il giudice polacco ha applicato la legge polacca anche alle statuizioni sul mantenimento. Il minore ha continuato a risiedere nello stesso Paese anche a seguito della decisione originaria, non rendendo possibile la modifica della legge applicabile.

Il caso esaminato mostra come il mutamento della legge applicabile può solo derivare da modifiche sostanziali di natura fattuale, in particolare dal cambio di residenza abituale del creditore (art. 3 comma 2 protocollo). Se tale circostanza non si verifica, la legge applicabile rimane la stessa anche nel contesto di una successiva modifica della decisione, in quanto il protocollo non consente un'applicazione indiscriminata del diritto sostanziale dello Stato di residenza abituale del creditore. ${ }^{84}$

Da ultimo, l'adesione al protocollo consente di beneficiare dell'abolizione dell'exequatur, come disciplinato dall'art. 17 e ss. Reg. n. 4/2009, consolidando ulteriormente il legame tra i due strumenti (vedi infra par. VIII). ${ }^{85}$

\section{Il coordinamento tra le fonti sulla legge applicabile}

16. Prima dell'entrata in vigore del Protocollo dell'Aia del 2007, la legge applicabile alle obbligazioni alimentari era regolata, tra gli Stati aderenti, dalla Convenzione dell'Aia del $1956^{86}$ e del 1973. ${ }^{87}$ Entrambe le convenzioni, come il protocollo, prevedono che la legge dello Stato di residenza abituale del creditore alimentare (del minore nel caso della convenzione più risalente) disciplini le obbligazioni alimentari.

Il rapporto tra il protocollo ed i precedenti strumenti internazionali è disciplinato dagli artt. 18 e 19 protocollo, oltre che dall'art. 69 Reg. n. 4/2009. Ai sensi dell'art. 18, il protocollo "sostituisce nei rapporti tra Stati contraenti» sia la Convenzione dell'Aia del 1956 che del 1973. Secondo l'art. 19, in assenza di dichiarazioni contrarie, il protocollo non pregiudica l'applicazione di quegli strumenti che riguardino le stesse materie e di cui uno Stato «è oppure sarà parte».

Infine, l'art. $69^{88}$ Reg. n. 4/2009 dichiara che l'applicazione delle convenzioni e degli accordi bilaterali o multilaterali non è pregiudicata dal regolamento (comma 1) ma che quest'ultimo, tuttavia,

\footnotetext{
${ }^{84}$ M. ANDRAE, «Einleitung HUntStProt», in Europäisches Zivilprozess cit., p. 645-647.

${ }^{85}$ V. LIPP, «Statut des Kindesunterhalts nach Aufenthaltswechsel», in Praxis des Internationalen Privat- und Verfahrensrechts, 2019, p. 402-403.

${ }^{86}$ Convenzione dell'Aia del 24 ottobre 1956 sulla legge applicabile alle obbligazioni alimentari nei confronti dei minori è reperibile sul sito www.hcch.net unicamente nella versione francese, in quanto adottata in tale lingua.

${ }^{87}$ Convenzione dell'Aia del 2 ottobre 1973 sulla legge applicabile alle obbligazioni alimentari, consultabile sul sito della Conferenza dell'Aia sia in lingua francese, sia in lingua inglese. Per un'analisi approfondita sugli strumenti internazionali in materia di legge applicabile alle obbligazioni alimentari e sui rapporti tra loro intercorrenti: L. CARBALLO PIÑEIRO, «Relationship between the 2007 Hague Maintenance Protocol and the Prior Hague Maintenance Conventions», in Planning the Future of Cross Border Families, a cura di I. Viarengo, F. C. Villata, Oxford and Portland, Oregon, Hart Publishing 2020, p. 385-396; K. Kroll-Ludwigs, «Das Verhältnis von Haager Unterhaltsprotokoll (2007) und Haager Unterhaltsübereinkommen (1973): lex posterior derogat legi priori?», in Praxis des Internationalen Privat- und Verfahrensrechts, 2016, p. 34-40; G. BADIALI, La disciplina internazionale degli obblighi alimentari, Napoli, 1994, p. 37 ss.; C. BISCARETTI DI RUFFIA, Le obbligazioni alimentari nel diritto internazionale privato convenzionale, Padova, Cedam 1989 p. 126 ss.; P. DE CesARI, «Le nuove convenzioni dell’Aja in materia di obbligazioni alimentari», in Rivista di diritto internazionale privato e processuale, 1983, p. $42 \mathrm{ss}$.

${ }^{88}$ Ai sensi dell'art. 69: «1. Il presente regolamento non pregiudica l'applicazione delle convenzioni e degli accordi bilaterali o multilaterali di cui uno o più Stati membri sono parte al momento dell'adozione del presente regolamento e che riguardano materie disciplinate dal presente regolamento, fatti salvi gli obblighi che incombono agli Stati membri in virtù dell'articolo 307
} 
prevale nelle relazioni tra Stati membri (comma 2). Tra i Paesi che vi hanno aderito, dunque, il protocollo viene applicato in luogo delle precedenti convenzioni in materia; l'applicazione degli strumenti internazionali, in linea di principio, non è pregiudicata né dal protocollo e né dal regolamento, ma quest'ultimo gode di applicazione prioritaria fra Stati membri.

L'applicazione di queste disposizioni si configura però problematica.

Particolarmente delicata è la questione relativa alla legge applicabile alle controversie tra Stati parte della Convenzione del 1956 o del 1973 che hanno aderito al protocollo e coloro che hanno adottato le suddette convenzioni ma non il protocollo. La Convenzione dell'Aia del 1956 continua ad essere applicata dal Liechtenstein e dalla Regione speciale di Macao (Cina) ${ }^{89}$ mentre quella del 1973 dall'Albania, dal Giappone, dalla Svizzera e dalla Turchia.

Infatti, l'art. 18 protocollo non indica esplicitamente se le suddette convenzioni continuino ad applicarsi nei confronti degli Stati che non sono parte del protocollo o se, data l'efficacia universale di quest'ultimo (art. 2), sia il protocollo a trovare applicazione..$^{90}$

Sul punto sono stati prospettati due opposti indirizzi. Un primo orientamento fa ancora riferimento alle convenzioni dell'Aia per determinare la legge applicabile nell'ipotesi in cui esse siano applicabili da entrambi gli Stati coinvolti. Infatti, il protocollo non può trovare applicazione fra i Paesi che non vi hanno aderito e, pertanto, questi non sostituisce le convenzioni dell'Aia come voluto dall'art. 18.

Inoltre, l'applicazione universale del protocollo non contrasta con l'art. 18, il quale rappresenta una deroga alla regola generale dell'art. 2; infatti, conformemente ai principi del diritto internazionale, un trattato continua a trovare applicazione fino a che lo stesso non sia sostituito o risolto unilateralmente. Questa evenienza non si è verificata, né con riferimento alla Convenzione dell'Aia del 1956, né a quella del 1973 e, pertanto, queste ultime trovano ancora applicazione in quegli Stati che non hanno aderito al protocollo, anche nei confronti di uno Stato che invece vi abbia aderito. ${ }^{91}$

Secondo questo primo indirizzo, ad esempio, in Germania ove la questione si propone frequentemente nei rapporti con la Svizzera e la Turchia, la legge applicabile viene determinata dalla Convenzione dell'Aia del 1973 nei confronti di creditori residenti in questi ultimi due Paesi. ${ }^{92}$ Quanto detto trova conferma negli artt. 69 Reg. n. 4/2009 e 19 comma 1 protocollo. ${ }^{93}$

Un orientamento differente ritiene invece di applicare il protocollo, in luogo delle convenzioni, basandosi sul carattere universale dello stesso, secondo cui le norme di conflitto ivi contenute trovano applicazione in maniera del tutto indipendente dal requisito della reciprocità ed anche laddove si tratti della legge di uno Stato non contraente. Secondo questo indirizzo, la Convenzione dell'Aia del 1973 viene sostituita anche nei rapporti tra Stati non contraenti, diversamente da quanto suggerisce la formu-

del trattato. 2. Nonostante il paragrafo 1 e fatto salvo il paragrafo 3, il presente regolamento prevale, tra gli Stati membri, sulle convenzioni e gli accordi che riguardano materie disciplinate dal presente regolamento e di cui sono parte gli Stati membri. 3. Il presente regolamento non osta all'applicazione della convenzione del 23 marzo 1962 tra la Svezia, la Danimarca, la Finlandia, l'Islanda e la Norvegia sul recupero dei crediti alimentari da parte degli Stati membri che ne sono parte, considerato che detta convenzione prevede, per quanto riguarda il riconoscimento, l'esecutività e l'esecuzione di decisioni: a) procedure semplificate e accelerate per l'esecuzione di decisioni in materia di alimenti; e b) disposizioni relative al patrocinio a spese dello Stato più favorevoli di quelle previste al capo $\mathrm{V}$ del presente regolamento. Tuttavia, l'applicazione di tale convenzione non priva il convenuto della tutela offertagli dagli articoli 19 e 21 del presente regolamento».

${ }^{89}$ La convenzione si applica esclusivamente alla regione amministrativa speciale di Macao poiché vi era in vigore a seguito di un'estensione fatta dal Portogallo, del quale era una colonia. Successivamente alla restituzione di Macao alla Repubblica popolare cinese (20 dicembre 1999), la Cina ha dichiarato che la convenzione continuerà ad applicarsi a Macao.

${ }^{90}$ F. PESCE, Le obbligazioni alimentari cit., p. 209-210.

${ }^{91}$ D. HeNRICH, Im Labyrinth des internationalen Unterhaltsrechts, in Zeitschrift für das gesamte Familienrecht, 2015, p. 1763.

${ }^{92}$ Cfr. BGB-Handkommentar mit AGG, EGBGB, ErbbauRG, LPartG, ProdhaftG, VBVG, VersAusglG, WEG und ausgewählten Rechtsquellen des IPR, Köln, Dr. Otto Schmidt 2017, Rn. 1; A. DutTA, «OLG Stuttgart: Anwendung des Haager Unterhaltsprotokolls in deutsch-türk. Fällen», in Zeitschrift für das gesamte Familienrecht, 2014, p. 2005-2006; M. ANDRAE, «Zum Beitritt der Europäischen Gemeinschaft zum Haager Protokoll über das Unterhaltskollisionsrecht», in GPR-Zeitschrift für das Privatrecht der Europäischen Union, 2010, p. 196.

${ }^{93}$ M. ANDRAE, «Kapitel III: Anwendbares Recht Art 15 EG-UntVO», in Europäisches Zivilprozess cit., p. 604-605. 
lazione dell'art. 18. Anche la Relazione esplicativa al protocollo curata da Andrea Bonomi ${ }^{94}$ propende per l'applicazione di quest'ultimo strumento. ${ }^{95}$

Pertanto, quando si tratta di un Paese che ha aderito al protocollo, la legge applicabile viene determinata da quest'ultimo e può portare anche all'applicazione del diritto sostanziale svizzero o turco, in quanto non è più imposto alcun obbligo di reciprocità tra Stati, come accadeva in vigenza degli strumenti internazionali precedenti. ${ }^{96}$ Quando la prospettiva è invece quella di un Paese che non ha aderito al protocollo, ma solo ad una delle convenzioni suddette, la legge applicabile viene determinata da queste ultime.

Infine, nel caso in cui nessuna delle suddette convenzioni sia stata oggetto di ratifica, trovano applicazione le norme di diritto internazionale privato di ciascun Paese.

17. La questione della legge applicabile alla Svizzera ed alla Turchia è talmente delicata che è significativo che le corti tedesche abbiano evitato di prendere un'esplicita posizione sul punto.

In un caso deciso dal Bundesgerichtshof ${ }^{97}$ il debitore (cittadino tedesco) e la creditrice (cittadina svizzera) concludono un contratto prematrimoniale di fronte ad un notaio tedesco. Nessuna indicazione viene data sulla legge applicabile al mantenimento. Le parti risiedono in Germania fino alla separazione; successivamente la creditrice si trasferisce col figlio in Svizzera. L'Amtsgericht Nürtingen applica il diritto tedesco e pronuncia lo scioglimento del vincolo matrimoniale delle parti, ponendo a carico del debitore il mantenimento della creditrice. In appello, l'Oberlandesgericht Stuttgart fonda la propria decisione sulla legge svizzera (legge dello Stato di residenza abituale della creditrice) sulla base dell'art. 2 protocollo ed incrementa l'assegno di mantenimento. Secondo il giudice, infatti, il fatto che la Svizzera non abbia aderito al protocollo non ha alcuna rilevanza nel caso di specie in quanto, grazie a carattere universale dello stesso, può trovare applicazione anche la legge di uno Stato non contraente.

Entrambe le parti ricorrono al Bundesgerichtshof. Questi considera privo di utilità indagare sull'operatività o meno del protocollo nei confronti della Svizzera in quanto ritiene che il diritto tedesco trovi applicazione in ogni caso. Infatti, sia sulla base dell'art. 8 Convenzione dell'Aia del $1973^{98}$ che dell'art. 5 protocollo, secondo cui nei rapporti tra «coniugi, ex coniugi o persone il cui matrimonio sia stato annullato» può applicarsi la legge dello Stato dell'ultima residenza abituale comune o di altra che presenti uno stretto collegamento, il diritto applicabile risulta quello tedesco. Nel primo caso, in quanto legge regolatrice del divorzio statuito dalle autorità tedesche; nel secondo, in virtù dell'opposizione del debitore al diritto svizzero in favore di quello dello Stato dell'ultima residenza comune delle parti.

Desta particolare interesse la parte finale della sentenza, ove il giudice di legittimità osserva di non poter porre fine al dibattito suddetto. Infatti il protocollo, a seguito della ratifica da parte dell'Unione europea, è soggetto unicamente all'interpretazione fornita dal giudice di Lussemburgo. ${ }^{99}$

Anche l'Oberlandesgericht Stuttgart ${ }^{100}$ nel caso relativo alla modifica del mantenimento in favore di due creditori residenti in Turchia affronta il problema della legge applicabile. Il giudice tedesco, accennando ai due indirizzi suddetti ma non schierandosi né in un senso né nell'altro, ritiene applicabile la legge turca al caso concreto, in quanto legge dello Stato di residenza abituale dei creditori, rilevante sia ai sensi della Convenzione dell'Aia del 1973 (ex art. 4) sia del protocollo (ex art. 3). L'Oberlandesgericht Stuttgart non prende posizione sul punto e si riferisce alla suddetta sentenza del Bundesgerichtshof, ove la questione è stata volutamente lasciata aperta.

Le citate sentenze mettono in luce l'esistenza di un problema applicativo che coinvolge ciascun operatore del diritto e non ha ancora trovato soluzione.

${ }^{94} \mathrm{Cfr}$. punto 199 della versione italiana consultabile sul sito https://assets.hcch.net/docs/a20ac557-f94d-42da-b625-776e7de69f01.pdf.

${ }^{95}$ N. CONTI, V. BISSMEIER, «Das neue Haager Unterhaltsprotokoll» cit., p. 63.

${ }^{96}$ R. HausmanN, «Ehegatten- und Kindesunterhalt», in Internationales und Europäisches Ehescheidungsrecht, a cura di R. Hausmann, München, C.H. Beck 2013.

${ }^{97}$ BGH 26 giugno 2013, in Praxis des Internationalen Privat- und Verfahrensrechts, 2014, p. 345-349.

${ }^{98}$ Secondo questa disposizione, la legge applicata al divorzio dovrebbe regolare sia le obbligazioni alimentari tra sposi, sia la modifica delle decisioni ad esse relative.

${ }^{99}$ Cfr. par. 37.

${ }^{100}$ OLG Stuttgart 17 gennaio 2014 cit., supra nota 30. 
Permane dunque, a quasi dieci anni dall' applicazione del protocollo, la necessità di un intervento chiarificatore della Corte di giustizia sul punto. Se quest'ultimo avrà meno rilevanza pratica nell'ipotesi in cui dall'applicazione di uno strumento o dall'altro consegua lo stesso risultato, lo stesso non può dirsi nel caso in cui la legge applicabile risulti invece differente.

Tralasciando in questa sede l'analisi della portata dell'obbligo di effettuare un rinvio pregiudiziale, si auspica che avanti ai giudici degli Stati membri giungano quanto prima contenziosi nei quali le norme di conflitto previste dal protocollo e dalla Convenzione dell'Aia del 1956 o 1973, non prescrivano l'applicazione della stessa legge.

Ad un tale risultato, come si è dimostrato nel corso degli anni, si fatica ad arrivare considerato il rapporto di continuità temporale e materiale che lega gli strumenti in esame.

\section{Il riconoscimento, l'esecutività e l'esecuzione dei provvedimenti in materia alimentare}

18. Il Reg. n. 4/2009 regola la circolazione delle decisioni fra Stati membri prevedendo, al capo IV, due distinti sistemi ed alcune norme comuni applicabili in entrambe le ipotesi.

Il primo sistema (ed il più innovativo) dispone il riconoscimento, senza possibilità di opposizione, nonché l'esecuzione ex lege di tutte quelle decisioni emesse in un Paese vincolato al Protocollo dell'Aia del 2007 (vedi supra par. VI). ${ }^{101}$

Il secondo rende invece necessaria, per gli Stati non aderenti al protocollo, una dichiarazione di esecutività tramite una procedura che si ispira a quella del previgente Reg. n. 44/2001. ${ }^{102}$ L'art. $48^{103}$ estende la disciplina del capo IV anche alle transazioni giudiziarie ed agli atti pubblici.

La prima ipotesi, tramite il riconoscimento e l'esecuzione automatica delle sentenze esecutive nello Stato di origine, comporta l'equiparazione di una decisione straniera ad una nazionale (artt. 17-22). Il convenuto che non sia comparso di fronte alle autorità giurisdizionali dello Stato membro di origine può però ottenere una dichiarazione di nullità della decisione emessa (cons. n. 29 e art. 19).

L'esecuzione della decisione può essere rifiutata dall'autorità giurisdizionale qualora il diritto ad ottenerla si sia prescritto ai sensi della legge dello Stato di origine o dello Stato richiesto (se più lungo), nonché qualora la decisione risulti inconciliabile con altra emessa in quest'ultimo. L'esecuzione può essere invece sospesa qualora nel Paese di origine sia stata presentata una domanda di riesame o l'esecutività della decisione sia stata sospesa (art. 21 comma 3). Tra i motivi di rifiuto o sospensione possono figurare anche quelli previsti dal diritto nazionale dello Stato dell'esecuzione ma, in ogni caso, il contrasto con l'ordine pubblico non determina il rigetto dell'esecuzione. ${ }^{104}$

Dall'art. 23 all'art. 38 viene invece disciplinata la procedura per il riconoscimento e l'esecuzione delle decisioni emesse nel Regno di Danimarca e nel Regno Unito, unici "Stati membri" non vincolati al protocollo.

Il riconoscimento è anche in questo caso automatico, a meno che non sussistano motivi di rifiuto previsti all'art. 24. Tra questi figurano, tra l'altro, la contrarietà all'ordine pubblico e l'incompatibilità della decisione con un'altra emessa nello Stato membro del riconoscimento. Una nuova decisione, che ne modifichi una precedente, non rientra invece nel concetto di «decisione inconciliabile» idonea a determinare il rifiuto del riconoscimento.

${ }^{101}$ R. PACIA, «Regolamento (CE) n. 4/2009: competenza, legge applicabile, riconoscimento ed esecuzione delle decisioni in materia di obbligazioni alimentari», in Studium Iuris, 2011, p. 15.

${ }^{102}$ M. C. Baruffi, «In tema di riconoscimento delle decisioni» cit., p. 136; M. Castellaneta, A. Leandro, «Il regolamento CE n. 4/2009» cit., p. 1090.

${ }_{103}$ Ai sensi dell'art. 48: «1. Le transazioni giudiziarie e gli atti pubblici esecutivi nello Stato membro d'origine sono riconosciuti in un altro Stato membro e hanno la stessa esecutività delle decisioni ai sensi del capo IV. 2. Le disposizioni del presente regolamento sono applicabili, se del caso, alle transazioni giudiziarie e agli atti pubblici. 3. L'autorità competente dello Stato membro d'origine rilascia, su istanza di qualsiasi parte interessata, un estratto della transazione giudiziaria o dell'atto pubblico utilizzando, a seconda dei casi, il modulo di cui agli allegati I e II ovvero agli allegati III e IV».

${ }^{104}$ F. Pocar, I. ViarenGO, «Il regolamento (CE) n. 4/2009» cit., p. 822-824. 
L'esecuzione di una decisione già esecutiva nel Paese di origine è disciplinata dalla lex fori di ciascuno Stato richiesto. La pronuncia deve essere dichiarata esecutiva presso quest'ultimo tramite un procedimento regolato dagli artt. 27-30.

La persona contro la quale si procede non può presentare alcuna osservazione in questa fase ma ha la facoltà di ricorrere contro la domanda volta ad ottenere la dichiarazione di esecutività (artt. $32 \mathrm{e}$ 33). Questa decisione può a sua volta essere oggetto di impugnazione, ma il rifiuto o la revoca, dipendono dai motivi di cui all'art. 24. ${ }^{105}$

Da quanto suesposto emerge che, al fine di dare esecuzione negli Stati membri alle decisioni provenienti dal Regno di Danimarca e dal Regno Unito, data l'assenza di norme di conflitto uniformi, sia prima necessario dichiararne l'esecutività; al contrario, le decisioni che provengono dagli altri Stati membri vengono automaticamente eseguite anche nei Paesi che non sono vincolati al protocollo. ${ }^{106}$

19. Le disposizioni del regolamento concernenti l'esecuzione delle decisioni hanno creato qualche problema applicativo, in quanto esse non individuano quale sia il giudice competente per questa fase.

La prassi ha cercato di colmare questo vuoto normativo applicando, in via suppletiva, l'art. 24 comma 5 Reg. n. 1215/2012, il quale attribuisce la competenza per la fase dell'esecuzione al giudice del luogo in cui questa avviene. ${ }^{107}$

La scelta di tale disposizione non appare però del tutto pertinente, in quanto le obbligazioni alimentari sono esplicitamente escluse dall' ambito di applicazione del Reg. n. 1215/2012 (art. 1 comma 2 lett. e). Infatti, dal 18 giugno 2011, la materia alimentare è disciplinata unicamente dal Reg. n. 4/2009, il quale rappresenta una lex specialis rispetto ai regolamenti che lo hanno preceduto.

Allo stesso tempo però, il Reg. n. 4/2009 disciplina la competenza internazionale solo per la fase di cognizione (artt. 3 e ss.); per quella di esecuzione invece, esso si limita a specificare che il procedimento è retto dalla legge dello Stato membro richiesto (art. 41 comma 1) ${ }^{108}$ senza indicare esplicitamente il giudice competente.

La questione è stata affrontata in tempi recenti dalla Corte di giustizia, adita dall'Amtsgericht Köln con rinvio pregiudiziale in data 14 gennaio $2019,{ }^{109}$ che ha chiarito in quali ipotesi l'opposizione all'esecuzione di una decisione straniera rientri nell'ambito di applicazione del Reg. n. 4/2009.

L'Amtsgericht Köln chiede infatti se un'opposizione all'esecuzione ai sensi dell'art. 767 ZPO, ${ }^{110}$ contro un titolo straniero in materia di alimenti, rientri nel campo di applicazione del Reg. n. 4/2009 o, in caso contrario, del Reg. n. 1215/2012 (art. 24 comma 5).

Nel 2009 il giudice polacco pone a carico del debitore, residente in Germania, il mantenimento di sua figlia, residente in Polonia, la quale, ottenuta l'apposizione della formula esecutiva dall'Amt-

\footnotetext{
${ }^{105}$ Ai sensi dell'art. 24: «Le decisioni non sono riconosciute: a) se il riconoscimento è manifestamente contrario all'ordine pubblico dello Stato membro in cui è richiesto il riconoscimento. Le norme sulla competenza non riguardano l'ordine pubbli$\mathrm{co}$; b) se la domanda giudiziale od un atto equivalente non è stato notificato o comunicato al convenuto contumace in tempo utile e in modo tale da consentirgli di presentare le proprie difese eccetto qualora, pur avendone avuto la possibilità, egli non abbia impugnato la decisione; c) se sono incompatibili con una decisione emessa tra le stesse parti nello Stato membro in cui è richiesto il riconoscimento; d) se sono incompatibili con una decisione emessa precedentemente tra le stesse parti in un altro Stato membro o in un paese terzo, in una controversia avente il medesimo oggetto e il medesimo titolo, qualora tale decisione soddisfi le condizioni necessarie per essere riconosciuta nello Stato membro in cui è richiesto il riconoscimento. La decisione che abbia l'effetto di modificare una precedente decisione in materia di obbligazioni alimentari a causa di un mutamento delle circostanze non è considerata una decisione inconciliabile ai sensi delle lettere c) o d)».

${ }^{106}$ M. NADEMLEINSKY, «Die neue EU-Unterhaltsverordnung» cit., p. 133.

${ }^{107}$ L'art. 24 comma 5, collocato nella sezione 6 del capo II del regolamento dispone che: «Indipendentemente dal domicilio delle parti, hanno competenza esclusiva le seguenti autorità giurisdizionali di uno Stato membro: (...) 5) in materia di esecuzione delle decisioni, le autorità giurisdizionali dello Stato membro nel cui territorio ha o ha avuto luogo l'esecuzione».

${ }^{108}$ L'art. 41 comma 1, intitolato «Procedimento e condizioni d'esecuzione» stabilisce che: «1. Fatte salve le disposizioni del presente regolamento, il procedimento d'esecuzione delle decisioni emesse in un altro Stato membro è disciplinato dalla legge dello Stato membro dell'esecuzione. Le decisioni emesse in uno Stato membro che sono esecutive nello Stato membro dell'esecuzione sono eseguite alle stesse condizioni delle decisioni emesse nello Stato membro dell'esecuzione»

${ }^{109}$ AG Köln 14 gennaio 2019, in Zeitschrift für Jugendhilfe und Familienrecht, 2019, p. 519-521.

${ }^{110}$ Ai sensi dell'art. 767 ZPO, intitolato "Vollstreckungsabwehrklage" (opposizione all'esecuzione), le contestazioni relative ad un credito accertato in sentenza devono essere sollevate davanti al giudice dell'esecuzione e sono ammissibili laddove $\mathrm{i}$ motivi siano sorti successivamente alla chiusura della discussione orale.
} 
sgericht Köln, avvia in Germania una procedura di esecuzione forzata contro il debitore. Quest'ultimo nell'aprile 2018, davanti allo stesso giudice, presenta un'opposizione all'esecuzione ai sensi dell'art. $767 \mathrm{ZPO}$, sostenendo di aver già effettuato il pagamento ed estinto il proprio debito.

Il giudice tedesco si interroga sulla propria competenza internazionale ad esaminare il merito dell'opposizione. Da una parte, infatti, se quest'ultima rientra nella materia delle obbligazioni alimentari, il Reg. n. 4/2009 sembrerebbe attribuire la competenza alle autorità polacche. L'opposizione presentata dal debitore equivale ad una modifica del titolo alimentare del quale si chiede l'esecuzione; per questa ragione deve applicarsi l'art. 8 Reg. n. 4/2009, il quale vieta al debitore che voglia ottenere la modifica di una decisione, di adire un giudice differente rispetto a quello che abbia statuito in origine, nell'ipotesi in cui il creditore continui a risiedere nello Stato in cui sia stata emessa la decisione. Il giudice del rinvio ritiene inoltre che solo in questo modo si preserva la posizione di favore del creditore sotto il profilo delle regole di competenza, in quanto si esclude che quest'ultimo si difenda da un'opposizione all'esecuzione, avente ad oggetto eccezioni di merito, in un foro differente da quello nel quale sia stata emessa la decisione.

Dall'altra parte, invece, l'art. 767 ZPO consente di sollevare le eccezioni relative al credito davanti al giudice dell'esecuzione e sembra quindi ritenere le autorità tedesche competenti a pronunciarsi sul punto. Tuttavia, secondo la dottrina tedesca, tale opposizione non rientra tra le azioni previste dal Reg. n. 4/2009, in quanto si riferisce alla fase di esecuzione, di cui il regolamento non disciplina la competenza.

Il giudice tedesco si chiede quindi se, esclusa l'applicazione del Reg. n. 4/2009, si possa qualificare l'opposizione all'esecuzione in conformità all'art. 24 comma 5 Reg. n. 1215/2012.

La Corte di giustizia, con sentenza Fx. c. Gz. del 4 giugno 2020, ${ }^{111}$ chiarisce che l'opposizione di un debitore all'esecuzione di un credito alimentare, che sia stato accertato dal giudice di uno Stato membro, rientra nell'ambito di applicazione del Reg. n. 4/2009 a condizione che presenti uno stretto collegamento con il procedimento di esecuzione.

L'eccezione di cui all'art. $767 \mathrm{ZPO}$, relativa all'adempimento del debito, non mira infatti a modificare la decisione originaria ma, piuttosto, a contestare l'importo da eseguire. Essa non viene neppure sollevata per ottenere un riesame della decisione e quindi, poiché tende esclusivamente ad impedirne l'esecuzione, lascia del tutto impregiudicato il titolo alimentare. In definitiva, con l'opposizione il debitore vuole solo ottenere l'esatta quantificazione dell'importo della decisione da eseguire.

Come osservato nell'opinione espressa dall'avvocato generale, l'esecuzione di una decisione rientra sempre nella competenza del giudice dello Stato membro dell'esecuzione, in quanto si tratta di un principio comune a tutte le misure di cooperazione in materia civile. Nei casi, dunque, in cui l'eccezione del debitore è intrinsecamente connessa al procedimento di esecuzione, la competenza a pronunciarsi nel merito dell'opposizione deve essere attribuita al giudice dello Stato membro in cui la decisione deve essere eseguita.

Questa circostanza viene confermata, secondo la Corte, dall'art. 41 comma 1 Reg. n. 4/2009 il quale, nell'indicare che la fase dell'esecuzione viene regolata dalla legge dello Stato dell'esecuzione, è anche norma attributiva di competenza per l'opposizione.

L'interpretazione contraria contrasterebbe con gli obiettivi di celerità e semplicità perseguiti dal regolamento; infatti, mentre la sentenza verrebbe eseguita nello Stato dell'esecuzione su istanza del creditore, il debitore sarebbe obbligato ad opporsi ad essa nello Stato in cui la decisione è stata emessa, facendo valere l'avvenuto adempimento dell'obbligazione accertata. L'impossibilità di opporsi nel Paese in cui viene eseguita la sentenza determinerebbe, dunque, un inutile dispendio di tempo e denaro per le parti e per l'apparato giudiziario degli Stati membri coinvolti.

\section{Le disposizioni transitorie}

20. L'applicazione del Reg. n. 4/2009 viene disciplinata dall'art. 75 . Il primo comma stabilisce infatti, «fatti salvi i paragrafi 2 e 3 », l'applicazione del regolamento ai procedimenti (transazioni giu-

${ }^{111}$ Corte di giustizia 4 giugno 2020, Fx c. Gz, 41/19. 
diziarie ed atti pubblici) instaurati dal 18 giugno 2011, data dalla quale ai sensi dell'art. 76 comma 3 si applica il regolamento.

Il comma secondo, invece, prevede l'applicazione delle disposizioni relative agli Stati membri non vincolati al protocollo qualora si richieda il riconoscimento e la dichiarazione di esecutività dopo la data di applicazione del Reg. n. 4/2009 per decisioni emanate anteriormente al 18 giugno 2011. L'exequatur è anche richiesto per le pronunce che rientrino, per quanto concerne il riconoscimento e l'esecuzione, nel campo di applicazione del Reg. n. 44/2001, laddove emanate successivamente al 18 giugno 2011 nell'ambito di procedimenti avviati anteriormente a questa data.

L'operatore del diritto che si trovi di fronte ad un credito alimentare deve per prima cosa verificare se questo rientri nel campo di applicazione del Reg. n. 44/2001 o del Reg. n. 4/2009. Laddove il credito venga qualificato come alimentare, ci si deve riferire a quest'ultimo strumento prestando però attenzione alla disciplina dell'art. $75 .{ }^{112}$

In un caso recente trattato dal Bundesgerichtshof ${ }^{13}$ il debitore ed i suoi figli minorenni litigano sulla necessità di una dichiarazione di esecutività di un provvedimento sul mantenimento. Nel dicembre 2011, la madre dei creditori presenta un'istanza al giudice polacco per ottenere il mantenimento provvisorio dei figli. Questo provvedimento viene chiesto nel corso del giudizio di divorzio, pendente in Polonia dal 2010. Anni dopo, i creditori chiedono che la decisione sia dichiarata esecutiva in Germania. Tale richiesta viene accolta in primo grado ma respinta in appello, sul presupposto che si applichi il regolamento e dunque la sentenza sia già esecutiva e non necessiti di exequatur. I creditori ricorrono al Bundesgerichtshof il quale conferma che il provvedimento polacco non necessita di exequatur.

Infatti, ai sensi dell'art. 17 comma 2 Reg. n. 4/2009, se una decisione proviene da uno Stato membro vincolato al Protocollo dell'Aia del 2007 ed è esecutiva nel Paese di origine, essa lo è automaticamente anche negli altri Stati membri. I creditori contestano invece l'applicabilità del Reg. n. 4/2009, in quanto la domanda di divorzio è stata introdotta precedentemente al 18 giugno 2011. Il Bundesgerichtshof, al contrario, dichiara che la domanda sul mantenimento per il periodo di svolgimento del giudizio di divorzio deve considerarsi autonoma e, poiché introdotta successivamente al 18 giugno 2011, soggetta all'applicazione del regolamento. Per tale ragione, il provvedimento polacco è esecutivo anche in Germania senza che sia necessaria alcuna dichiarazione in tal senso.

Nella sentenza in esame si discute della necessità di una dichiarazione di esecutività di una decisione straniera. Problema centrale è l'applicabilità del Reg. n. 4/2009, ed in particolare delle disposizioni in materia di riconoscimento ed esecuzione delle sentenze. Ai sensi dell'art. 76 comma 3, il regolamento si applica dal 18 giugno 2011 ed ai sensi dell'art. 75 comma 1, a tutti i procedimenti avviati successivamente a questa data. L'art. 75 comma 2, al contrario, indica due ipotesi nelle quali si rende ancora necessario l'exequatur. Il momento in cui viene instaurata una controversia per l'ottenimento di un titolo alimentare ne determina la disciplina applicabile e, nell'ipotesi in cui se ne chieda poi l'esecuzione, la necessità o meno di una dichiarazione di esecutività.

L'art. 3 lett. c) nelle azioni relative allo stato delle persone, quali ad esempio quelle sul divorzio, consente al giudice di statuire anche sulla domanda inerente le obbligazioni alimentari quando accessoria all'azione principale. La possibilità di portare di fronte allo stesso giudice la domanda di divorzio e quella relativa al mantenimento non può però incidere sull'autonomia delle stesse, ciascuna delle quali, qualora introdotta in un momento diverso, sarà collocata in un proprio spazio temporale.

${ }^{112}$ C. Kern, «Die Vollstreckbarkeit ausländischer familienrechtlicher Zahlungstitel», in Praxis des Internationalen Privatund Verfahrensrechts., 2017, p. 475-476.

${ }^{113}$ BGH 25 settembre 2019, in Zeitschrift für das gesamte Familienrecht, 2020, p. 123-125. 